\title{
Axion-modified photon propagator, Coulomb potential, and Lamb shift
}

\author{
S. Villalba-Chávez, ${ }^{*}$ A. Golub, ${ }^{\dagger}$ and C. Müller \\ Institut für Theoretische Physik I, Heinrich-Heine-Universität Düsseldorf, \\ Universitätsstraße 1, 40225 Düsseldorf, Germany
}

(Received 28 June 2018; published 5 December 2018)

\begin{abstract}
A consistent renormalization of a quantum theory of axion-electrodynamics requires terms beyond the minimal coupling of two photons to a neutral pseudoscalar field. This procedure is used to determine the self-energy operators of the electromagnetic and the axion fields with an accuracy of second-order in the axion-diphoton coupling. The resulting polarization tensor is utilized for establishing the axion-modified Coulomb potential of a static pointlike charge. In connection, the plausible distortion of the Lamb-shift in hydrogenlike atoms is established and the scopes for searching axionlike particles in high-precision atomic spectroscopy and in experiments of Cavendish-type are investigated. Particularly, we show that these hypothetical degrees of freedom are ruled out as plausible candidates for explaining the proton radius anomaly in muonic hydrogen. A certain loophole remains, though, which is linked to the nonrenormalizable nature of axion-electrodynamics.
\end{abstract}

DOI: 10.1103/PhysRevD.98.115008

\section{INTRODUCTION}

That the path integral measure in quantum chromodynamics (QCD) is not invariant under an axial chiral $\mathrm{U}(1)_{\mathrm{A}}$-transformation provides a clear evidence that this classical symmetry does not survive the quantization procedure. As a consequence of this anomaly, QCD should not be a charge-parity $(C P)$-preserving framework. However, with astonishing experimental accuracy, no $C P$ violation event is known within the theory of the strong interactions. This so-called "strong $C P$-problem" finds a consistent theoretical solution by postulating a global $\mathrm{U}(1)_{\mathrm{PQ}}$-invariance in the standard model $(\mathrm{SM})$, which compensates the $C P$-violating term via its spontaneous symmetry breaking [1]. While this mechanism seems to be the most simple and robust among other possible routes of explanation, it is accompanied by a new puzzle linked to the nonobservation of the associated Nambu-Goldstone boson, i.e., the QCD axion [2,3]. As a consequence, constraints resulting from this absence indicate a feeble interplay between this hypothetical particle and the well-established SM branch, rendering its detection a very challenging problem to overcome. Still, various

\footnotetext{
*selym@tp1.uni-duesseldorf.de

†Alina.Golub@uni-duesseldorf.de

*c.mueller@tp1.uni-duesseldorf.de
}

Published by the American Physical Society under the terms of the Creative Commons Attribution 4.0 International license. Further distribution of this work must maintain attribution to the author(s) and the published article's title, journal citation, and DOI. Funded by SCOAP. experimental endeavors are currently oriented to detect this elusive degree of freedom or, more generally, an associated class of particle candidates sharing its main features, i.e., axionlike particles (ALPs). Some of them being central pieces in models which attempt to explain the dark matter abundance in our Universe [4-8], whereas others are remnant features of string compactifications [9-12].

The problematic associated with the ALPs detection demands both to exploit existing high-precision techniques and to develop new routes along which imprints of these hypothetical particles can be observed $[13,14]$. Descriptions of the most popular detection methods can be found in Refs. [15-19]. A vast majority of these searches relies on the axion-diphoton coupling encompassed within axionelectrodynamics [20]. Correspondingly, many photonrelated experiments such as those searching for light shining through a wall [21-28] and the ones based on polarimetry detections [29-33] have turned out to be particularly powerful.

Contrary to that, precision tests of the Coulomb's law via atomic spectroscopy and experiments of Cavendishtype have not been used so far in the search for ALPs, although they are known to constitute powerful probes for other well-motivated particle candidates [34-37]. In particular, these setups provide the best laboratory bounds on minicharged particles in the sub $\mu \mathrm{eV}$ mass range. Simultaneously, by investigating the role of ALPs in atomic spectra, one might elucidate whether the quantum vacuum of these hypothetical degrees of freedom may be the source for the large discrepancy $>5 \sigma$ between the proton radius that follows from the Lamb-shift in muonic hydrogen 
versus the established value based on electron scattering and the Lamb-shift in ordinary hydrogen [38-41]. Various theoretical investigations have been put forward seeking for a satisfactory explanation for this anomaly [42-46], some of them including hypothetical scalar particles.

Against the background of these circumstances, it is relevant to derive modifications of the Coulomb potential due to quantum vacuum fluctuations of axionlike fields and to study their potential consequences. The former are encompassed in the corresponding vacuum polarization tensor whose calculation, however, is not a straightforward task as far as axion quantum electrodynamics $\left(\mathrm{QED}_{\mathrm{A}}\right)$ is concerned. This is because it requires-first of all-a meaningful implementation of the corresponding perturbative expansion in this nonrenormalizable framework. In analogy to quantum gravity [47-53], the expansion in terms of the axion-diphoton coupling gives rise to an infinite number of divergencies that cannot be reabsorbed in the renormalization constants associated with the parameters and fields of the theory. Unless a similar amount of counterterms is added, this feature spoils the predictivity of the corresponding scattering matrix, preventing the construction of a consistent quantum theory of axion-electrodynamics. Hence, the perturbative renormalizability of this theory demands unavoidably the incorporation of higher dimensional operators. This, in turn, comes along with the presence of a large number of free parameters which have to be fixed from experimental data. It is, however, known that this formal aspect relaxes because many of these higherdimensional terms are redundant, in that the ultimate scattering matrix is not sensitive to their coupling constants [54-57]. As a matter of fact, all contributions of this nature can be formally eliminated while the effective Lagrangian acquires counterterms which allow for the cancellation of the loop divergences. Besides, when working at a certain level of accuracy, only a finite number of counterterms is needed and the cancellations of the involved infinities can be carried out pretty much in the same way as in conventional renormalizable field theories.

In this paper, axion-electrodynamics is regarded as a Wilsonian effective theory parametrizing the leading order contribution of an ultraviolet completion linked to physics beyond the SM. Its quantization is used for determining the self-energy operator of the electromagnetic field with an accuracy of second-order in the axion-diphoton coupling. This result is utilized then for obtaining the modified Coulomb potential of a static pointlike charge. In connection, the plausible distortion of the Lamb-shift in hydrogenlike atoms is established. Particular attention is paid to a limitation caused by the nonrenormalizable feature of axion electrodynamics which prevents us from having a precise and clear picture of the axion physics at distances smaller than the natural cutoff imposed by the axion-diphoton coupling. In contrast to previous studies of the Lamb-shift involving minicharged particles and hidden photon fields, this property introduces an unknown uncertainty that cannot be determined, unless the ultraviolet completion of axion-electrodynamics is found. We argue that — up to this uncertainty-axionlike particles are ruled out as plausible candidates for explaining the proton radius anomaly in muonic hydrogen $\left(\mathrm{H}_{\mu}\right)$. Parallelly, spectroscopic results linked to a variety of transitions in hydrogen are exploited to probe the sensitivity of this precision technique in the search for ALPs. We show that, as a consequence of the mentioned feature, high-precision spectroscopy lacks of sufficient sensitivities as to improve the existing laboratory constraints on the parameter space of ALPs.

Our treatment is organized as follows. Firstly, in Secs. II $\mathrm{A}$ and II B, techniques known from effective field theories are exploited for establishing the vacuum polarization tensor within an accuracy of the second order in the axion-diphoton coupling. There we show that, despite the electrically neutral nature of ALPs, the polarization tensor closely resembles the one obtained in QED. The similarity is stressed even further in Secs. III A and III B, where various asymptotes of the polarization tensor are established and the general expression for the axionCoulomb potential is determined. The latter outcome is presented in such a way that a direct comparison with the Uehling potential can be carried out. Also in Sec. III B, we derive the corresponding modification to the Lamb-shift and emphasize the problematic introduced by both the effective scenario and the use of atomic $s$-states. In Sec. III C we give some estimates and discuss the advantages and disadvantages of testing ALPs via excited states in $\mathrm{H}_{\mu}$, whereas in Sec. IV our conclusions are exposed. Some details about the particleghost content of the theory are provided in Appendix A. Finally, in Appendix B the sensitivity levels associated with precision tests of the axion-modified Coulomb law via experiments of Cavendish-type are presented.

\section{THE MODIFIED PHOTON PROPAGATOR IN AXION-ELECTRODYNAMICS}

\section{A. Effective field theory approach}

Axion-electrodynamics relies on an effective action characterized by a natural ultraviolet scale $\Lambda_{\mathrm{UV}}$ at which the $\mathrm{U}(1)_{\mathrm{PQ}}$-symmetry is broken spontaneously. It combines the standard Maxwell Lagrangian, the free Lagrangian density of the pseudoscalar field $\bar{\phi}(x)$ and an interaction term coupling two photons and an axion. Explicitly,

$$
\begin{aligned}
S_{\bar{g}}= & \int d^{4} x\left\{-\frac{1}{4} f_{\mu \nu} f^{\mu \nu}+\frac{1}{2} \partial_{\mu} \bar{\phi} \partial^{\mu} \bar{\phi}\right. \\
& \left.-\frac{1}{2} \bar{m}^{2} \bar{\phi}^{2}+\frac{1}{4} \bar{g} \bar{\phi} \tilde{f}_{\mu \nu} f^{\mu \nu}\right\} .
\end{aligned}
$$

Here, $f_{\mu \nu}=\partial_{\mu} \bar{a}_{\nu}-\partial_{\nu} \bar{a}_{\mu}$ stands for the electromagnetic field tensor, whereas its dual reads $\tilde{f}^{\mu \nu}=\frac{1}{2} \epsilon^{\mu \nu \alpha \beta} f_{\alpha \beta}$ with 
$\epsilon^{0123}=1$. Hereafter, we use a metric with signature $\operatorname{diag}\left(g_{\mu \nu}\right)=(1,-1,-1,-1)$, and a unit system in which the speed of light, the Planck constant and the vacuum permitivity are set to unity, $c=\hbar=\epsilon_{0}=1$. As the axiondiphoton coupling $\bar{g}=\Lambda_{\mathrm{UV}}^{-1}$ has an inverse energy dimension, this effective theory belongs to the class of perturbatively nonrenormalizable frameworks. In the following we will suppose that $\Lambda_{\mathrm{UV}}$ is a very large parameter in order to extend integrals over the momentum components to an infinitevolume Fourier space. Whenever no problem of convergence arises, the integrals over the spacetime coordinates will also be extended to the whole Minkowski space.

We want to use Eq. (1) to derive radiative corrections up to second-order in the axion-diphoton coupling $\sim \bar{g}^{2}$. For this, we shall use well-established effective field theory techniques which have been applied extensively within the context of quantum gravity [47-53] and chiral perturbation theory [58-61] (see also Refs. [62-64] for their application in nonlinear QED). In connection, we will suppose that $S_{\bar{g}}$ characterizes - at energies substantially lower than $\Lambda_{\mathrm{UV}}$ the leading order contribution of its UV-completion which is Lorentz and gauge invariant. Hence, all higherdimensional operators which are consistent with these fundamental symmetries should be included in Eq. (1), implying that an infinite number of counterterms is necessary to cancel out the divergences linked to oneparticle irreducible Feynman diagrams [65]. However, to extract quantitative predictions from the radiative corrections in the second-order approach in the axion-diphoton coupling, it is sufficient to incorporate the next-to-leading order term of $S_{\bar{g}}$. Combining the described method with a dimensional analysis, we find that the renormalization of the self-energy operators in $\mathrm{QED}_{\mathrm{A}}$ should be handled by two local operators of dimension 6:

$$
\begin{aligned}
S_{\bar{g}^{2}}= & \int d^{4} x\left\{\frac{1}{2} \bar{g}^{2} \overline{\mathfrak{b}}_{a}^{2}\left(\partial_{\mu} f^{\mu \lambda}\right)\left(\partial_{\nu} f_{\lambda}^{\nu}\right)\right. \\
& \left.+\frac{1}{2} \bar{g}^{2} \overline{\mathfrak{b}}_{\phi}^{2}\left(\partial_{\mu} \bar{\phi}\right) \square\left(\partial^{\mu} \bar{\phi}\right)+\cdots\right\} .
\end{aligned}
$$

Various local operators sharing both the symmetry of the theory and the same dimensionality can be found. However, it can be easily verified that all of them reduce to those given in Eq. (2) through integrations by parts. The Wilson parameters $\overline{\mathfrak{b}}_{a, \phi}$ determine the strength of the contributions above. They might be determined by a matching procedure provided the UV-completion of $S_{\bar{g}}$ is known. Since we ignore the precise form of the latter, they will be considered as arbitrary. We emphasize that the appearance of the square of the Wilson parameters in $S_{\bar{g}^{2}}$ guarantees that—at least at tree level — the theory is causal [i.e., free of tachyons; see also discussion below].

It is worth remarking that the action resulting from the combination of Eqs. (1) and (2) cannot be considered as an ordinary action containing higher-order derivatives. Within a quantum effective theory approach, higher-dimensional operators-like those exhibited in Eq. (2) - are suppressed by higher powers of $\bar{g}$, so that their consequences at low energies relative to $\Lambda_{\mathrm{UV}}=\bar{g}^{-1}$ are tiny when compared with the effects resulting directly from $S_{\bar{g}}[65,66]$. To all effects, they must be treated as perturbations, otherwise a violation of the unitarity takes place due to the occurrence of Pauli-Villars ghosts. While this problem has been noted in previous studies (see for instance [67,68] and references therein), it is instructive to review it once again in the present context. To this end, we first note that the appearance of ghosts emerges quite straightforwardly when investigating the axion Green function that results from combining the corresponding kinetic term and the second line of Eq. (2):

$$
G\left(p^{2}\right)=\frac{1}{p^{2}-\bar{m}^{2}}-\frac{1}{p^{2}-\bar{m}_{\mathrm{s}}^{2}} .
$$

At the pole $p=\bar{m}^{2}$, the residue of this Green function is $\left.\operatorname{Res} G\left(p^{2}\right)\right|_{p^{2}=\bar{m}^{2}}=1,{ }^{1}$ whereas at $p^{2}=\bar{m}_{\mathrm{s}}^{2}=\left(\bar{g} \overline{\mathfrak{b}}_{\phi}\right)^{-2}$ it turns out to be $\left.\operatorname{Res} G\left(p^{2}\right)\right|_{p^{2}=\bar{m}_{\mathrm{s}}^{2}}=-1$. Hence, the vacuum excitations linked to the former pole have a positive definite norm in the Hilbert space, as should correspond to asymptotically single-particle states. Conversely, the square of the norm associated with the remaining massive excitations is nonpositive and no physical state can be associated with them (ghost states), leading parallely to a violation of unitarity [67]. Noteworthy, as the associated higher-dimensional operator [see Eq. (2)] contains the square of $\overline{\mathfrak{b}}_{\phi}$, the ghost mass is real and its fictitious propagation does not involve a speed faster than the speed of light. The term written in the first line of Eq. (2) leads to a similar scenario, ${ }^{2}$ but with a different ghost mass $\bar{m}_{\mathrm{gh}}^{2}=\left(\bar{g} \overline{\mathfrak{b}}_{a}\right)^{-2}$ [for details, see Appendix A]. The described situation provides evidences that symmetry arguments are not enough to obtain a well-behaved quantum theory of the fields involved in $\mathcal{S}=S_{\bar{g}}+S_{\bar{g}^{2}}$. However, if the scattering matrix linked to its UV-completion is unitary, it is natural to expect that the one associated with $\mathcal{S}$, covering quantum processes at lower energies $\ll \Lambda_{\mathrm{UV}}$, is unitary too. This idea justifies the restriction given above Eq. (3). We remark that these "ghost-providing" contributions are redundant operators which can be dropped from the effective Lagrangian without changing observables [54-56]. Later on, this outcome is used to remove them

\footnotetext{
${ }^{1}$ Although the exposition is made in terms of bare parameters, the idea extends straightforwardly when renormalized quantities are considered instead.

${ }^{2}$ The origin of the electromagnetic theory that results from combining the Maxwell theory with the higher-derivative operator written in the first line of Eq. (2) dates back to the work of P. Podolsky [69]. For further developments see Refs. [70-77].
} 
conveniently while the effective Lagrangian acquires counterterms which allow for the cancellation of the divergences associated with the loops that are calculated here [see below Eq. (7)].

Now, to carry out the renormalization program, the set of "bare" quantities $\left(\bar{m}, \bar{g}, \bar{m}_{\mathrm{gh}}, \bar{m}_{\mathrm{s}}, \bar{a}_{\mu}, \bar{\phi}\right)$ should be replaced by the respective renormalized parameters $\left(m, g, m_{\mathrm{gh}}, m_{\mathrm{s}}\right.$, $\left.a_{\mathrm{R}}^{\mu}, \phi_{\mathrm{R}}\right)$. In connection, each term in $\mathcal{S}=S_{\bar{g}}+S_{\bar{g}^{2}}+\cdots$ has to be parametrized by a renormalization constant so that the action to be considered from now on is

$$
\begin{aligned}
\mathcal{S}= & \mathcal{S}_{\mathrm{R}}+\mathcal{S}_{\mathrm{ct}}+\cdots, \\
\mathcal{S}_{\mathrm{R}}= & \int d^{4} x\left\{-\frac{1}{4} f_{\mathrm{R}}^{2}+\frac{1}{2}\left(\partial \phi_{\mathrm{R}}\right)^{2}-\frac{1}{2} m^{2} \phi_{\mathrm{R}}^{2}\right. \\
& \left.+\frac{1}{4} g \phi_{\mathrm{R}} \tilde{f}_{\mathrm{R}} f_{\mathrm{R}}+\frac{1}{2 m_{\mathrm{gh}}^{2}}\left(\partial f_{\mathrm{R}}\right)^{2}-\frac{1}{2 m_{\mathrm{s}}^{2}} \phi_{\mathrm{R}} \square^{2} \phi_{\mathrm{R}}\right\}, \\
\mathcal{S}_{\mathrm{ct}}= & \int d^{4} x\left\{-\frac{1}{4}\left(\mathcal{Z}_{3}-1\right) f_{\mathrm{R}}^{2}+\frac{1}{2}\left(\mathcal{Z}_{\phi}-1\right)\left(\partial \phi_{\mathrm{R}}\right)^{2}\right. \\
& -\frac{1}{2} m^{2}\left(\mathcal{Z}_{m}-1\right) \phi_{\mathrm{R}}^{2}+\frac{1}{2 m_{\mathrm{gh}}^{2}}\left(\mathcal{Z}_{\mathrm{gh}}-1\right)\left(\partial f_{\mathrm{R}}\right)^{2} \\
& \left.+\frac{1}{4}\left(\mathcal{Z}_{g}-1\right) g \phi_{\mathrm{R}} \tilde{f}_{\mathrm{R}} f_{\mathrm{R}}-\frac{1}{2 m_{\mathrm{s}}^{2}}\left(\mathcal{Z}_{\mathrm{s}}-1\right) \phi_{\mathrm{R}} \square^{2} \phi_{\mathrm{R}}\right\} .
\end{aligned}
$$

Here, $f_{\mathrm{R}} \equiv f_{\mathrm{R}}^{\mu \nu}=\partial^{\mu} a_{\mathrm{R}}^{\nu}-\partial^{\nu} a_{\mathrm{R}}^{\mu}$ is the renormalized electromagnetic tensor with $a_{\mathrm{R}}^{\mu}(x)=\mathcal{Z}_{3}^{-1 / 2} \bar{a}^{\mu}(x)$. Likewise, the renormalized axion field $\phi_{\mathrm{R}}(x)$ and its bare counterpart $\bar{\phi}(x)$ are connected via $\phi_{\mathrm{R}}(x)=\mathcal{Z}_{\phi}^{-1 / 2} \bar{\phi}(x)$, where $\mathcal{Z}_{\phi}$ is the corresponding wavefunction renormalization constant. Any other bare parameter relates to its respective renormalized quantity following multiplicative renormalizations according to

$$
\begin{gathered}
\bar{m}=m \sqrt{\frac{\mathcal{Z}_{m}}{\mathcal{Z}_{\phi}}}, \quad \bar{g}=g \frac{\mathcal{Z}_{g}}{\mathcal{Z}_{3} \sqrt{\mathcal{Z}_{\phi}}}, \\
\bar{m}_{\mathrm{gh}}=m_{\mathrm{gh}} \sqrt{\frac{\mathcal{Z}_{3}}{\mathcal{Z}_{\mathrm{gh}}}}, \quad \bar{m}_{\mathrm{s}}=m_{\mathrm{s}} \sqrt{\frac{\mathcal{Z}_{\phi}}{\mathcal{Z}_{\mathrm{s}}}} .
\end{gathered}
$$

It is worth remarking that in Eq. (4) the shorthand notations $f_{\mathrm{R}}^{2} \equiv f_{\mathrm{R} \mu \nu} f_{\mathrm{R}}^{\mu \nu}, \tilde{f}_{\mathrm{R}} f_{\mathrm{R}} \equiv \tilde{f}_{\mathrm{R} \mu \nu} f_{\mathrm{R}}^{\mu \nu},\left(\partial \phi_{\mathrm{R}}\right)^{2} \equiv\left(\partial_{\mu} \phi_{\mathrm{R}}\right)\left(\partial^{\mu} \phi_{\mathrm{R}}\right)$ have been used. When inserting the expressions for the ghost masses [see below Eq. (3)] in those relations given in the second line of Eq. (5), we link the bare and renormalized Wilsonian parameters:

$$
\overline{\mathfrak{b}}_{a}=\mathfrak{b}_{a} \frac{1}{\mathcal{Z}_{g}} \sqrt{\mathcal{Z}_{3} \mathcal{Z}_{\mathrm{gh}} \mathcal{Z}_{\phi}}, \quad \overline{\mathfrak{b}}_{\phi}=\mathfrak{b}_{\phi} \frac{\mathcal{Z}_{3}}{\mathcal{Z}_{g}} \sqrt{\mathcal{Z}_{\mathrm{s}}}
$$

where the connection between $\bar{g}$ and $g$ has been used. Noteworthy, within our second-order approximation in the coupling constant, no modification on the renormalized axion-diphoton coupling $g$ can be expected, i.e., $\mathcal{Z}_{g}$ would not deviate from its classic tree-level value $\mathcal{Z}_{g}=1$. Hence, from now on, no distinction between the renormalized and physical coupling is needed. However, we emphasize that its bare counterpart $\bar{g}$ is still subjected to a renormalization due to the wave functions renormalization constants $\mathcal{Z}_{3}$ and $\mathcal{Z}_{\phi}$.

At the quantum level, the dynamical information of the system described by Eq. (4) is rooted within the Green functions. They can be obtained from the generating functional

$$
Z[j, \dot{\mathrm{i}}]=\frac{\int \mathcal{D} \phi_{\mathrm{R}} \mathcal{D} a_{\mathrm{R}} \mathrm{e}^{i \mathcal{S}+i \int d^{4} x\left[-\frac{1}{2 \zeta}\left(\partial_{\mu} a_{\mathrm{R}}^{\mu}\right)^{2}+a_{\mathrm{R}}^{\mu} \mathrm{i}_{\mu}+\phi_{\mathrm{R}} j\right]}}{\int \mathcal{D} \phi_{\mathrm{R}} \mathcal{D} a_{\mathrm{R}} \mathrm{e}^{i \mathcal{S}}},
$$

where $j(x)$ and $\mathrm{j}^{\mu}(x)$ denote the external currents associated with the axion $\phi_{\mathrm{R}}(x)$ and the gauge field $a_{\mathrm{R}}^{\mu}(x)$. The contribution in the exponent of Eq. (7) which is proportional to the parameter $\frac{1}{\zeta}$ guarantees a covariant quantization of $a_{\mathrm{R}}^{\mu}(x)$. At this point it turns out to be convenient to bring the renormalized Lagrangian in $\mathcal{S}_{\mathrm{R}}$ [see Eq. (4)] to a canonical form in which the terms linked to the PauliVillars ghosts are dropped. This can be achieved by performing the following local field redefinitions within the path integral [see Eq. (7)]:

$$
\phi_{\mathrm{R}} \rightarrow \phi_{\mathrm{R}}-\frac{\square}{2 m_{\mathrm{s}}^{2}} \phi_{\mathrm{R}}, \quad a_{\mathrm{R}}^{\mu} \rightarrow a_{\mathrm{R}}^{\mu}-\frac{\square}{2 m_{\mathrm{gh}}^{2}} a_{\mathrm{R}}^{\mu},
$$

and by keeping the accuracy to the order $g^{2}$. As these transformations are linear in $\phi_{\mathrm{R}}(x)$ and $a_{\mathrm{R}}^{\mu}(x)$, the associated Jacobian leads to a decoupling between the corresponding Fadeev-Popov ghosts and the fundamental fields. The equivalence theorem [54,56] generalizes this fact by dictating that no change is induced on the scattering matrix through shifts of this nature; still they modify the initial parameters of the theory. Indeed, in our problem the redefinition of $\phi_{\mathrm{R}}(x)$ leads to a kinetic term of the form $\frac{1}{2}\left(1-\frac{m^{2}}{m_{\mathrm{s}}^{2}}\right)\left(\partial \phi_{\mathrm{R}}\right)^{2}$. The additional factor contained in this expression can be reabsorbed in $\mathcal{S}_{\mathrm{ct}}$ [see Eq. (4)] by redefining the wavefunction renormalization constant for the axion field $\mathcal{Z}_{\phi}-\frac{m^{2}}{m_{s}^{2}} \rightarrow \mathcal{Z}_{\phi}^{\prime}$. Similarly, we redefine $\mathcal{Z}_{\text {gh }}-\mathcal{Z}_{3} \rightarrow \mathcal{Z}_{\text {gh }}^{\prime}, \mathcal{Z}_{\mathrm{s}}-\mathcal{Z}_{\phi} \rightarrow \mathcal{Z}_{\mathrm{s}}^{\prime}, \mathcal{Z}_{3} \rightarrow \mathcal{Z}_{3}^{\prime}$ and $\mathcal{Z}_{m} \rightarrow \mathcal{Z}_{m}^{\prime}$ to reabsorb terms arising when transforming $\mathcal{S}_{\mathrm{ct}}$. Therefore, apart from this unobservable effect, the Pauli-Villars ghosts have no result other than to remove the divergences that might arise from the respective one-particle irreducible graphs.

\section{B. Renormalized photon propagator in a modified minimal subtraction scheme}

We pursue our investigation by determining the modification to the photon propagator $D_{\alpha \beta}^{(0)}(x, \tilde{x})$ due to quantum vacuum fluctuations of a pseudoscalar axion field $\phi_{\mathrm{R}}(x)$. 


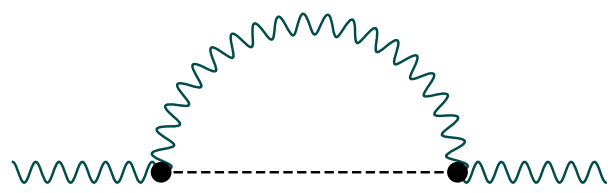

FIG. 1. Feynman diagram depicting the axion-modified vacuum polarization tensor. While the dashed line represents the free axion propagator, the internal wavy line denotes the free photon propagator $D_{\mu \nu}^{(0)}(x, \tilde{x})$. Here, the external wavy lines represent amputated photon legs.

The use of Eq. (7) allows us to express the modified photon propagator $D_{\alpha \beta}(x, \tilde{x})$ as

$$
D_{\alpha \beta}(x, \tilde{x})=\left.\frac{1}{i^{2}} \frac{\delta^{2} Z[j, \dot{\mathrm{j}}]}{\delta \dot{\mathbf{l}}^{\alpha}(x) \delta \dot{\mathbf{j}}^{\beta}(\tilde{x})}\right|_{j, \mathbf{i}=0} .
$$

We then expand Eq. (7) up to the order $g^{2}$ and insert the resulting expression into the formula above. As a consequence, the corrected photon propagator reads

$$
\begin{aligned}
D_{\alpha \beta}(x, \tilde{x})= & D_{\alpha \beta}^{(0)}(x, \tilde{x})+\int d^{4} y d^{4} \tilde{y} D_{\alpha \mu}^{(0)}(x, y) \\
& \times i \Pi^{\mu \nu}(y, \tilde{y}) D_{\nu \beta}^{(0)}(\tilde{y}, \tilde{x})+\mathcal{O}\left(g^{4}\right) .
\end{aligned}
$$

Here $\Pi^{\mu \nu}(y, \tilde{y})$ encompasses the expression for the unrenormalized polarization tensor [see Fig. 1] as well as counterterms that allow for the cancellation of the divergences associated with this loop. Analytically, it reads

$$
\begin{aligned}
\Pi^{\mu \nu}(y, \tilde{y})= & i g^{2} \epsilon^{\mu \alpha \epsilon \tau} \epsilon^{\nu \beta \sigma \rho}\left[\partial_{\sigma}^{\tilde{y}} \partial_{\epsilon}^{y} \Delta_{\mathrm{F}}^{(0)}(\tilde{y}, y)\right]\left[\partial_{\tau}^{y} \partial_{\rho}^{\tilde{y}} D_{\alpha \beta}^{(0)}(y, \tilde{y})\right] \\
& +\left\{\mathcal{Z}_{3}^{\prime}-1+\frac{\square}{m_{\mathrm{gh}}^{2}}\left(\mathcal{Z}_{\mathrm{gh}}^{\prime}-1\right)\right\} \\
& \times\left[\square g^{\mu \nu}-\partial^{\mu} \partial^{\nu}\right] \delta^{4}(y-\tilde{y}),
\end{aligned}
$$

where $\Delta_{\mathrm{F}}^{(0)}(x, \tilde{x})=\int d^{4} p \frac{i}{p^{2}-m^{2}+i 0} \mathrm{e}^{i p(x-\tilde{x})}$, with $d^{4} p \equiv$ $d^{4} p /(2 \pi)^{4}$ refers to the unperturbed ALP propagator, whereas $D_{\alpha \beta}^{(0)}(x, \tilde{x})=\int d^{4} p \frac{-i q_{\alpha \beta}}{p^{2}+i 0} \mathrm{e}^{i p(x-\tilde{x})}$ denotes the photon propagator in Feynman gauge $[\zeta=1]$. It is worth remarking that, in momentum space the polarization tensor $\Pi^{\mu \nu}\left(p_{1}, p_{2}\right)=\int d^{4} x d^{4} \tilde{x} \mathrm{e}^{-i p_{1} x} \Pi^{\mu \nu}(x, \tilde{x}) e^{i p_{2} \tilde{x}}$ reads

$$
\begin{array}{r}
\Pi^{\mu \nu}\left(p_{1}, p_{2}\right)=\delta_{p_{1}, p_{2}}\left\{i g^{2} \epsilon^{\mu \tau \alpha \beta} \epsilon^{\nu \sigma \gamma \rho} g_{\sigma \tau} p_{2 \beta} p_{2 \rho} \mathscr{K}_{\alpha \gamma}\right. \\
\left.-\left[\mathcal{Z}_{3}^{\prime}-1-\frac{p_{2}^{2}}{m_{\mathrm{gh}}^{2}}\left(\mathcal{Z}_{\mathrm{gh}}^{\prime}-1\right)\right]\left(p_{2}^{2} q^{\mu \nu}-p_{2}^{\mu} p_{2}^{\nu}\right)\right\},
\end{array}
$$

where the shorthand notation $\delta_{p_{1}, p_{2}} \equiv(2 \pi)^{4} \delta^{4}\left(p_{1}-p_{2}\right)$ has been introduced and

$$
\mathscr{K}_{\alpha \gamma}=\int \frac{d^{4} q q_{\alpha} q_{\gamma}}{q^{2}\left[\left(q-p_{2}\right)^{2}-m^{2}\right]}
$$

which diverges quadratically as $|q| \rightarrow \infty$. The regularization of $\Pi_{\mu \nu}\left(p_{1}, p_{2}\right)$ is then carried out by using a standard Feynman parametrization $\left[\frac{1}{a b}=\int_{0}^{1} \frac{d s}{[b+(a-b) s)^{2}}\right]$ and by continuing the loop integral to $D=4-\epsilon, \epsilon \rightarrow 0^{+}$dimensions via the replacement

$$
\int d^{4} q \ldots \rightarrow(\mathcal{C} \mu)^{\epsilon} \int d^{D} q \ldots
$$

where $\mathcal{C}=e^{\frac{1}{2}(\gamma-1)} /(4 \pi)^{1 / 2}$ and $\gamma=0.5772 \ldots$ is the EulerMascheroni constant. In this context, $\mu$ denotes a dimensionful parameter, i.e., the substracting point that follows when rescaling the renormalized axion-diphoton coupling $g \rightarrow g \mu^{\epsilon / 2}$ in $D$-dimensions so that its mass dimension -1 is kept. Also, when going from four to $D$-dimensions, the Wilsonian parameters [see Eqs. (2) and (6)] rescale $\mathfrak{b}_{i} \rightarrow \mathfrak{b}_{i} \mu^{-\epsilon / 2}$ with $i=a, \phi$ while their dimensionless feature is retained.

Now, we integrate over $q$ and Taylor expand the resulting expression in $\epsilon$. As a consequence, Eq. (12) becomes

$$
\begin{aligned}
\Pi^{\mu \nu}\left(p_{1}, p_{2}\right)= & \delta_{p_{1}, p_{2}}\left(\mathcal{g}^{\mu \nu} p_{2}^{2}-p_{2}^{\mu} p_{2}^{\nu}\right) \pi\left(p_{2}^{2}\right), \\
\pi\left(p^{2}\right)= & \frac{g^{2}}{16 \pi^{2}} \int_{0}^{1} d s \Delta(s)\left[\frac{2}{\epsilon}-\ln \left(\frac{\Delta(s)}{\mu^{2}}\right)\right] \\
& -\left(\mathcal{Z}_{3}^{\prime}-1\right)+\frac{p^{2}}{m_{\mathrm{gh}}^{2}}\left(\mathcal{Z}_{\mathrm{gh}}^{\prime}-1\right)
\end{aligned}
$$

with $\Delta(s)=m^{2} s-p^{2} s(1-s)$. Manifestly, the term associated with the factor $\epsilon^{-1}$ is singular as $\epsilon \rightarrow 0$. In contrast to QED, such a divergence cannot be reabsorbed fully in the wavefunction renormalization constant of the electromagnetic field $\mathcal{Z}_{3}^{\prime}$ by enforcing that the radiative correction should not alter the residue of the photon propagator at $p^{2}=0[65,78]$. We solve this problem, by choosing the counterterms in the following form

$$
\mathcal{Z}_{3}^{\prime}-1=\lim _{\epsilon \rightarrow 0} \frac{g^{2} m^{2}}{16 \pi^{2} \epsilon}, \quad \mathcal{Z}_{\mathrm{gh}}^{\prime}-1=\lim _{\epsilon \rightarrow 0} \frac{g^{2} m_{\mathrm{gh}}^{2}}{48 \pi^{2} \epsilon} .
$$

Notice that the ratio of scales $m g$ acts like a dimensionless coupling constant. Thus, the one-loop renormalized polarization tensor in a modified minimal subtraction scheme $(\overline{\mathrm{MS}})$ scheme reads

$$
\begin{aligned}
\Pi_{\overline{\mathrm{MS}}}^{\mu \nu}\left(p_{1}, p_{2}\right) & =\delta_{p_{1}, p_{2}}\left[p_{2}^{2} q^{\mu \nu}-p_{2}^{\mu} p_{2}^{\nu}\right] \pi_{\overline{\mathrm{MS}}}\left(p_{2}^{2}\right), \\
\pi_{\overline{\mathrm{MS}}}\left(p^{2}\right) & =-\frac{g^{2}}{16 \pi^{2}} \int_{0}^{1} d s \Delta(s) \ln \left(\frac{\Delta(s)}{\mu^{2}}\right) .
\end{aligned}
$$


Noteworthy, this expression satisfies the transversality condition $p_{1 \mu} \Pi_{\overline{\mathrm{MS}}}^{\mu \nu}\left(p_{1}, p_{2}\right)=\Pi_{\overline{\mathrm{MS}}}^{\mu \nu}\left(p_{1}, p_{2}\right) p_{2 \mu}=0$.

Some comments are in order. First, when the QED action is extended with those terms belonging to $\mathrm{QED}_{\mathrm{A}}$, the expression for $\mathcal{Z}_{3}^{\prime}-1$ found in Eq. (16) will be added to the corresponding one-loop QED-expression $\left[\mathcal{Z}_{3(\mathrm{QED})}\right]$. $^{3}$ This operation allows us to define the standard renormalized $\mathrm{U}(1)$-charge as usual $e_{\mathrm{R}}=\tilde{Z}_{3(1 \text { loop })}^{1 / 2} \bar{e}$, with the bare charge $\bar{e}$ and $\tilde{\mathcal{Z}}_{3(1 \text { loop })}=\mathcal{Z}_{3}^{\prime}+\mathcal{Z}_{3(\mathrm{QED})}$. Finally, taking into account Eqs. (16) and (17), the Fourier transform of Eq. (10) is, up to an unessential longitudinal term,

$$
\begin{aligned}
D_{\overline{\mathrm{MS}}}^{\mu \nu}\left(p_{1}, p_{2}\right) & =\delta_{p_{1}, p_{2}} D_{\overline{\mathrm{MS}}}^{\mu \nu}\left(p_{2}\right), \\
D_{\overline{\mathrm{MS}}}^{\mu \nu}(p) & =\frac{-i q^{\mu \nu}}{p^{2}}\left[1+\pi_{\overline{\mathrm{MS}}}\left(p^{2}\right)\right] .
\end{aligned}
$$

This formula constitutes the starting point for further considerations. In the next section it will be used to establish the axion-modified Coulomb potential.

\section{Axion self-energy operator, renormalized mass vs physical mass}

Our aim in this section is to determine the axion selfenergy operator. Its associated Feynman diagram is depicted in Fig. 2. This object encloses the way in which the quantum vacuum fluctuations of the electromagnetic field correct the axion propagator. To show this analytically, we expand the generating functional for the Green function [see Eq. (7)] up to first order in $g^{2}$. Once this step has been carried out, the resulting expression is twice differentiated functionally with respect to the axion source $j(x)$ leading to

$$
\begin{aligned}
\Delta_{\mathrm{F}}(x, \tilde{x})= & \left.\frac{1}{i^{2}} \frac{\delta^{2} Z[j, \dot{\mathrm{j}}]}{\delta j(x) \delta j(\tilde{x})}\right|_{j, \mathrm{i}=0} \\
= & \Delta_{\mathrm{F}}^{(0)}(x, \tilde{x})-\int d^{4} y d^{4} \tilde{y} \Delta_{\mathrm{F}}^{(0)}(x, y) \\
& \times i \Sigma(y, \tilde{y}) \Delta_{\mathrm{F}}^{(0)}(\tilde{y}, \tilde{x})+\mathcal{O}\left(g^{4}\right) .
\end{aligned}
$$

Here, $\Sigma(y, \tilde{y})$ comprises the expression of the unrenormalized axion self-energy operator as well as some possible counterterms. Explicitly,

$$
\begin{aligned}
\Sigma(y, \tilde{y})= & -\frac{i}{2} g^{2} \epsilon^{\mu \nu \tau \sigma} \epsilon^{\alpha \beta \rho \gamma}\left[\partial_{\sigma}^{y} \partial_{\gamma}^{\tilde{y}} D_{\mu \alpha}^{(0)}(y, \tilde{y})\right] \\
& \times\left[\partial_{\tau}^{y} \partial_{\rho}^{\tilde{y}} D_{\beta \nu}^{(0)}(\tilde{y}, y)\right]-\left\{\square\left(\mathcal{Z}_{\phi}^{\prime}-1\right)\right. \\
& \left.+\frac{\square^{2}}{m_{\mathrm{s}}^{2}}\left(\mathcal{Z}_{\mathrm{s}}^{\prime}-1\right)+m^{2}\left(\mathcal{Z}_{m}^{\prime}-1\right)\right\} \delta^{4}(y-\tilde{y}) .
\end{aligned}
$$

\footnotetext{
${ }^{3}$ An explicit expression for $\mathcal{Z}_{3(\mathrm{QED})}$, in dimensional regularization, can be found in Eq. (19.37) of Ref. [65].
}

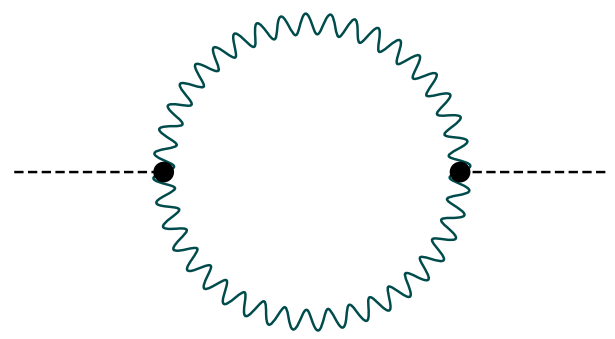

FIG. 2. Diagrammatic representation of the axion self-energy operator mediated by quantum vacuum fluctuations of the electromagnetic field. In contrast to Fig. 1, the external dashed lines represent amputated ALPs legs, whereas the internal wavy lines represent photon propagators $D_{\mu \nu}^{(0)}(x, \tilde{x})$.

Next, we Fourier transform $\Sigma(y, \tilde{y})$ and regularize its divergent integral via dimensional regularization as made in Sec. II B. However, in contrast to the case treated there, the associated divergence at $\epsilon \rightarrow 0$ is fully reabsorbed here in a renormalization constant

$$
\mathcal{Z}_{\mathrm{s}}^{\prime}-1=\lim _{\epsilon \rightarrow 0} \frac{g^{2} m_{\mathrm{s}}^{2}}{32 \pi^{2} \epsilon}
$$

whereas $\mathcal{Z}_{m}^{\prime}$ does not deviate from its classic tree-level value $\left[\mathcal{Z}_{m}^{\prime}=1\right]$. This feature extends beyond the one-loop approximation because the axion-photon vertex prevents the proliferation of self-interacting terms for ALPs containing no derivatives [66]. Despite this, the bare mass $\bar{m}$ still is subject to a finite renormalization due to the axion wavefunction renormalization constant [see Eq. (5)], which does not deviate from the classical value $\mathcal{Z}_{\phi}^{\prime}=1$. Keeping in mind all these details, we find that-in a $\overline{\mathrm{MS}}$ schemethe renormalized axion self-energy operator is given by

$$
\begin{aligned}
\Sigma_{\overline{\mathrm{MS}}}\left(p_{1}, p_{2}\right) & =\delta_{p_{1}, p_{2}} \Sigma_{\overline{\mathrm{MS}}}\left(p_{2}^{2}\right), \\
\Sigma_{\overline{\mathrm{MS}}}\left(p^{2}\right) & =\frac{3 g^{2} p^{2}}{32 \pi^{2}} \int_{0}^{1} d s \Delta_{m \rightarrow 0}(s) \ln \left(\frac{\Delta_{m \rightarrow 0}(s)}{\mu^{2}}\right),
\end{aligned}
$$

where $\Delta_{m \rightarrow 0}(s)=-p^{2} s(1-s)$ [see below Eq. (15)]. As we could have anticipated, this expression is independent of the renormalized axion mass. It is, perhaps, worth stressing that-in a $\overline{\mathrm{MS}}$-scheme- the square of the physical mass $m_{\text {phy }}^{2}$ is the value of $p^{2}$ for which the real part of the two-points irreducible function ${ }^{4}$ :

$$
\begin{gathered}
\Gamma\left(p_{1}, p_{2}\right)=\delta_{p_{1}, p_{2}}\left[p_{2}^{2}-m_{\text {phy }}^{2}\right], \\
m_{\text {phy }}^{2}=m^{2}-\Sigma_{\overline{\mathrm{MS}}}\left(m_{\mathrm{phy}}^{2}\right)
\end{gathered}
$$

vanishes. Whenever the subtracting parameter satisfies $\mu \gg m_{\text {phy }} \exp \left[-32 \pi^{2} /\left(g^{2} m_{\text {phy }}^{2}\right)\right], m_{\text {phy }} \approx m$ holds. Hence,

\footnotetext{
${ }^{4}$ This expression can be established from the identity $\int d^{4} q \Gamma\left(p_{1}, q\right) \Delta_{\mathrm{F}}\left(q, p_{2}\right)=-i \delta_{p_{1}, p_{2}}$, where $\Delta_{\mathrm{F}}\left(q, p_{2}\right)$ stands for the Fourier transform of Eq. (19).
} 
the expression for the polarization tensor [see Eq. (17)] as a function of $m_{\text {phy }}^{2}$ would not differ from the one given in terms of the renormalized mass.

At this point we find it interesting to make a comparison between Eq. (22) and the polarization tensor given in Eq. (17). To this end, it is convenient to reexpress the latter as follows:

$$
\begin{aligned}
\Pi_{\overline{\mathrm{MS}}}^{\mu \nu}\left(p_{1}, p_{2}\right) & =\delta_{p_{1}, p_{2}} \varkappa_{\overline{\mathrm{MS}}}\left(p^{2}\right)\left[g^{\mu \nu}-\frac{p_{2}^{\mu} p_{2}^{\nu}}{p_{2}^{2}}\right], \\
\varkappa_{\overline{\mathrm{MS}}}\left(p^{2}\right) & =p^{2} \pi_{\overline{\mathrm{MS}}}\left(p^{2}\right) .
\end{aligned}
$$

In this formula, $\varkappa_{\overline{\mathrm{MS}}}\left(p^{2}\right)$ represents the only nontrivial eigenvalue of the polarization tensor [79,80], which-in the limit under consideration - turns out to be smaller than $\Sigma_{\overline{\mathrm{MS}}}\left(p^{2}\right)$ by a factor $-2 / 3$.

Let us finally remark that, in addition to the axiondiphoton interplay, axion self-coupling [81] as well as effective interactions with electron, proton and neutron might occur [16,82-84]. In such a case further one-loop contributions to the axion self-energy operator might arise. However, these contributions depend on coupling constants other than the one mediating the interaction between an axion and two photons.

\section{AXION-COULOMB POTENTIAL}

\section{A. Screening of the electric charge and finite renormalization: Setting the subtracting parameter}

Hypothetical distortions of Coulomb's law can always be determined through the temporal component of the electromagnetic four-potential (hereafter, to simplify notation, $\mathfrak{a}^{\mu}(x)$ has to be understood as $\left.a_{\mathrm{R}}^{\mu}(x)\right)$

$$
\begin{aligned}
\mathfrak{a}^{\alpha}(x) & =-i \int D_{\overline{\mathrm{MS}}}^{\alpha \beta}(x, \tilde{x}) j_{\beta}(\tilde{x}) d^{4} \tilde{x}, \\
D_{\overline{\mathrm{MS}}}^{\alpha \beta}(x, \tilde{x}) & =\int d^{4} p_{1} d^{4} p_{2} e^{i p_{1} x} D_{\overline{\mathrm{MS}}}^{\alpha \beta}\left(p_{1}, p_{2}\right) e^{-i p_{2} \tilde{x}}
\end{aligned}
$$

where $D_{\overline{\mathrm{MS}}}^{\alpha \beta}\left(p_{1}, p_{2}\right)$ is given in Eq. (18). Here $j^{\beta}(\tilde{x})=$ $\mathfrak{q} \delta_{0}^{\beta} \delta^{3}(\tilde{x})$ denotes the four-current density of a pointlike static charge $\mathfrak{q}$ placed at the origin $\tilde{\boldsymbol{x}}=\mathbf{0}$ of our reference frame. Particularizing the expression above for $\alpha=0$, we end up with

$$
\mathfrak{a}_{0}(\boldsymbol{x})=\mathfrak{q} \int \frac{d^{3} p}{\boldsymbol{p}^{2}}\left[1+\pi_{\overline{\mathrm{MS}}}\left(\boldsymbol{p}^{2}\right)\right] e^{-i p \cdot \boldsymbol{x}}
$$

At this point it is worth emphasizing that, while the expression for $\pi_{\overline{\mathrm{MS}}}\left(\boldsymbol{p}^{2}\right)$ [see Eq. (17)] is finite, its dependence on the subtracting point $\mu$ introduces an arbitrariness. To remove it, we consider the expression of the electrostatic energy between two electrons in momentum space $\mathcal{U}(\boldsymbol{p})$. It can be established easily by taking the integrand above, with $\mathfrak{q} \rightarrow e_{\mathrm{R}}$. After multiplying the resulting expression by $e_{\mathrm{R}}$, we find

$$
\mathcal{U}(\boldsymbol{p})=-e_{\mathrm{R}} \mathfrak{a}_{0}(\boldsymbol{p})=-\frac{e_{\mathrm{scr}}^{2}(\boldsymbol{p})}{\boldsymbol{p}^{2}},
$$

where the screened charge $e_{\mathrm{scr}}^{2}(\boldsymbol{p})=e_{\mathrm{R}}^{2}\left[1+\pi_{\overline{\mathrm{MS}}}\left(\boldsymbol{p}^{2}\right)\right]$ has been defined.

As we still have freedom of performing finite renormalizations, we can demand that $\pi_{\overline{\mathrm{MS}}}\left(\boldsymbol{p}^{2}\right)$ vanishes as $|\boldsymbol{p}| \rightarrow 0$. Since the corresponding length scale $|\boldsymbol{x}| \rightarrow \infty$, $e_{\text {scr }}^{2}(|x| \rightarrow \infty)$ can be identified with the electrostatic charge that is measured in experiments at low energies. This natural renormalization condition $\left[\pi_{\overline{\mathrm{MS}}}(\mathbf{0})=0\right]$ holds for the subtracting parameter $\mu=m \exp [-1 / 4]$. The renormalized polarization tensor then reads

$$
\begin{aligned}
\Pi_{\mathrm{R}}^{\alpha \beta}\left(p_{1}, p_{2}\right)= & \delta_{p_{1}, p_{2}}\left[p_{2}^{2} q^{\alpha \beta}-p_{2}^{\alpha} p_{2}^{\beta}\right] \pi_{\mathrm{R}}\left(p_{2}^{2}\right), \\
\pi_{\mathrm{R}}\left(p^{2}\right)= & -\frac{g^{2} m^{2}}{64 \pi^{2}}\left[1-\frac{1}{3} \frac{p^{2}}{m^{2}}\right] \\
& -\frac{g^{2}}{16 \pi^{2}} \int_{0}^{1} d s \Delta(s) \ln \left(\frac{\Delta(s)}{m^{2}}\right),
\end{aligned}
$$

whereas the axion self-energy operator [see Eq. (22)] reduces to

$$
\begin{aligned}
\Sigma_{\mathrm{R}}\left(p_{1}, p_{2}\right) & =\delta_{p_{1}, p_{2}} \Sigma_{\mathrm{R}}\left(p_{2}^{2}\right), \\
\Sigma_{\mathrm{R}}\left(p^{2}\right) & =-\frac{g^{2} p^{4}}{64 \pi^{2}}\left[\ln \left(-\frac{p^{2}}{m^{2}}\right)-\frac{7}{6}\right] .
\end{aligned}
$$

The results obtained so far are summarized in Fig. 3, which displays the behavior of $\pi_{\mathrm{R}}\left(p^{2}\right)$ [left panel] and $\Sigma_{\mathrm{R}}\left(p^{2}\right)$ [right panel] as a function of $p^{2} / \mathrm{m}^{2}$. In both panels the respective real and imaginary parts are shown in green and red, manifesting by themselves the non-Hermitian feature of the polarization tensor and the axion self-energy operator. To support this numerical evaluation from an analytic viewpoint, we first determine an exact expression for the imaginary parts. To this end, we restore the $i 0$-prescription $\left[m^{2} \rightarrow m^{2}-i 0\right]$ in Eq. (28) and apply the formula $\ln (-\mathcal{A}-i 0)=\ln (|\mathcal{A}|)-i \pi$ with $\mathcal{A}>0$. Explicitly,

$$
\begin{aligned}
& \operatorname{Im}\left[\pi_{\mathrm{R}}\left(p^{2}\right)\right]=-\frac{g^{2} p^{2}}{96 \pi}\left[1-\frac{m^{2}}{p^{2}}\right]^{3} \Theta\left(p^{2}-m^{2}\right), \\
& \operatorname{Im}\left[\Sigma_{\mathrm{R}}\left(p^{2}\right)\right]=\frac{g^{2}\left(p^{2}\right)^{2}}{64 \pi} \Theta\left(p^{2}-m^{2}\right),
\end{aligned}
$$

where $\Theta(x)$ denotes the unit step function. We note that for an on-shell photon $\left[p^{2}=0\right]$, the imaginary part of $\Pi_{\mathrm{R}}^{\mu \nu}$ vanishes, which implies-according to the optical theorem - that the emission of an ALP from a photon accompanied by the radiation of another photon is forbidden. This fact agrees with the outcome resulting 

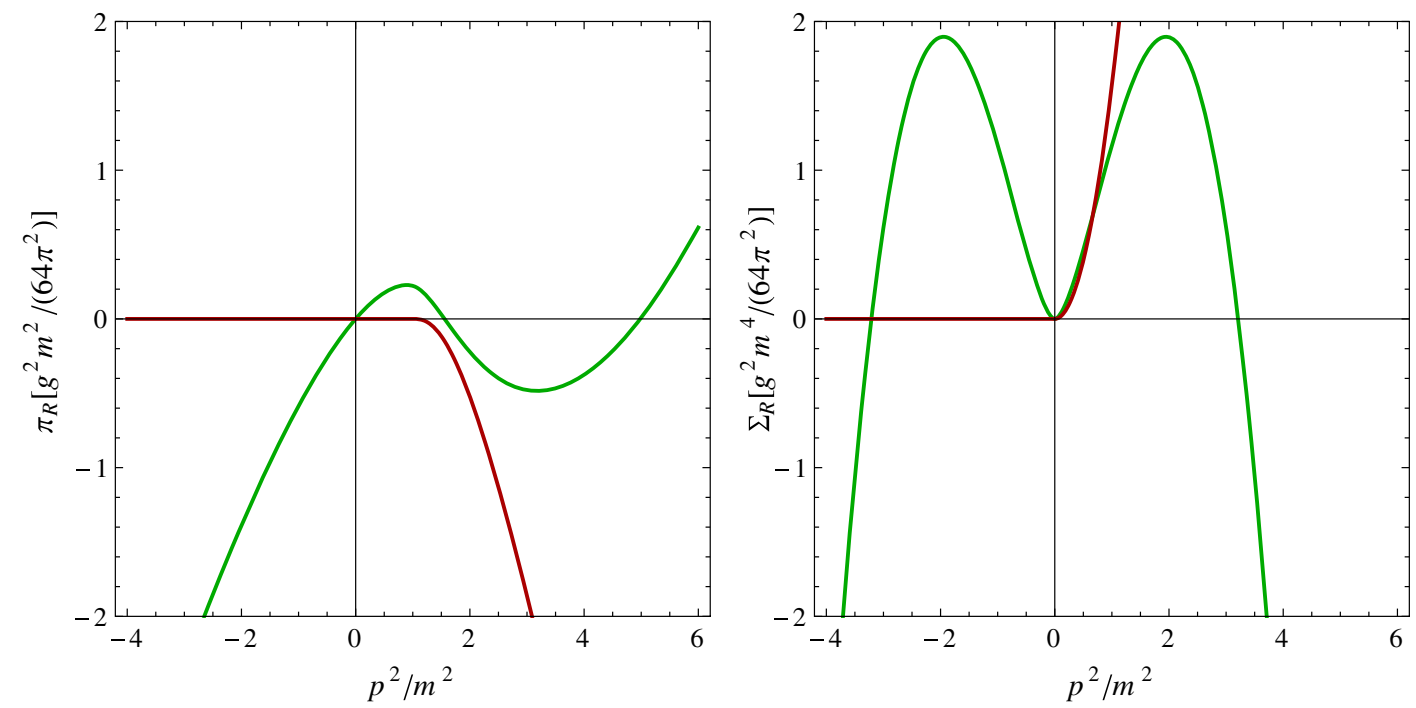

FIG. 3. In the left panel, the behavior of the form factor of the vacuum polarization tensor $\pi_{\mathrm{R}}\left(p^{2}\right)$ [see Eq. (28)] is shown as a function of $p^{2} / m^{2}$. The right panel depicts the corresponding dependence of the axion self-energy operator $\Sigma_{\mathrm{R}}\left(p^{2}\right)$ [see Eq. (29)]. The respective real and imaginary parts are displayed in green and red.

from an analysis of the corresponding energy-momentum balance.

The expression for the imaginary part of the one-loop self-energy operator $\operatorname{Im}\left[\Sigma_{\mathrm{R}}\left(p^{2}\right)\right]$ [second line in Eq. (30)] coincides with the result found previously in Ref. [85] through a direct application of the cutting rules. Its on-shell evaluation $\left[p^{2}=m^{2}\right]$ should allow us to determine the total rate of the decay process $\phi \rightarrow \gamma \gamma$ via the relation $\operatorname{Im}\left[\Sigma_{\mathrm{R}}\left(m^{2}\right)\right]=m \Gamma_{\phi \rightarrow \gamma \gamma}$, provided the optical theorem is valid. With accuracy to first order in $g^{2}$, this formula is indeed verified because an expression for $\Gamma_{\phi \rightarrow \gamma \gamma}$-relying on the corresponding $S$-matrix amplitude-can be inferred directly from the corresponding neutral pion decay rate [see for instance Eq. (19.119) in Ref. [86]]. This fact evidences that the unitarity is preserved, at least within the second order approximation in the axion-diphoton coupling $g$.

Further asymptotic expressions of Eq. (28) are elucidated. For $m^{2} \gg p^{2}$, we find that $\pi_{\mathrm{R}}\left(p^{2}\right)$ approaches to

$$
\pi_{\mathrm{R}}\left(p^{2} \ll m^{2}\right) \approx \frac{g^{2} p^{2}}{144 \pi^{2}}\left[1-\frac{3}{8} \frac{p^{2}}{m^{2}}\right] .
$$

Conversely, for $\left|p^{2}\right| \gg m^{2}$, its asymptotic behavior turns out to be dominated by the following function

$$
\left.\pi_{\mathrm{R}}\right|_{\left|p^{2}\right| \gg m^{2}} \approx \frac{g^{2} p^{2}}{96 \pi^{2}}\left[\ln \left(\frac{\left|p^{2}\right|}{m^{2}}\right)-i \pi \Theta\left(p^{2}\right)-\frac{7}{6}\right] .
$$

Notably, when $p$ is timelike $\left[p^{2}>0\right]$, the expression above gets an imaginary contribution $\operatorname{Im}\left[\pi_{\mathrm{R}}\left(p^{2} \gg m^{2}\right)\right] \approx$ $-g^{2} p^{2} /(96 \pi)$, which coincides with the leading order term of Eq. (30) when the condition $p^{2} \gg m^{2}$ is considered.

\section{B. Electrostatic potential and modified Lamb-shift}

The first contribution in Eq. (26) can be integrated straightforwardly, leading to the unperturbed Coulomb potential $\mathfrak{a}_{C}(\boldsymbol{x})=\mathfrak{q} /(4 \pi|\boldsymbol{x}|)$. The second one, on the other hand, will be computed by using $\pi_{\mathrm{R}}\left(\boldsymbol{p}^{2}\right)$ rather than $\pi_{\overline{\mathrm{MS}}}\left(\boldsymbol{p}^{2}\right)$. With all these details in mind we write

$$
\begin{aligned}
\mathfrak{a}_{0}(\boldsymbol{x}) & =\mathfrak{a}_{C}(\boldsymbol{x})+\delta \mathfrak{a}(\boldsymbol{x}), \\
\delta \mathfrak{a}(\boldsymbol{x}) & =\mathfrak{q} \int \frac{d^{3} p}{\boldsymbol{p}^{2}} \pi_{\mathrm{R}}\left(\boldsymbol{p}^{2}\right) e^{-i \boldsymbol{p} \cdot \boldsymbol{x}} .
\end{aligned}
$$

For evaluating $\delta \mathfrak{a}(\boldsymbol{x})$ explicitly, it is convenient to integrate by parts in Eq. (28) and use an equivalent representation of $\pi_{\mathrm{R}}\left(\boldsymbol{p}^{2}\right)$ instead:

$$
\pi_{\mathrm{R}}\left(p^{2}\right)=\frac{g^{2} p^{2}}{144 \pi^{2}}\left[1-\frac{3}{2} p^{2} \int_{0}^{1} \frac{d s s^{3}}{m^{2}-p^{2}(1-s)}\right] .
$$

Observe that, for applying this formula in Eq. (33), $p_{0}$ must be set to zero. Taking this into account, the integral over the momentum can be carried out with relative ease. After developing the change of variable $u=1 /(1-s)^{1 / 2}$, the axion-modified potential turns out to be

$$
\mathfrak{a}_{0}(\boldsymbol{x})=\frac{\mathfrak{q}}{4 \pi|\boldsymbol{x}|}\left\{1+\frac{g^{2} m^{2}}{48 \pi^{2}} \int_{1}^{\infty} \frac{d u}{u^{5}}\left[u^{2}-1\right]^{3} e^{-m|x| u}\right\} .
$$


We remark that spurious contributions containing Dirac deltas $\delta^{3}(x)$ have been ignored since the theory is predictive only for distances $|\boldsymbol{x}| \gg g$. Although the integral involved in this formula can be calculated analytically, we will keep it as it stands. Mainly, because it will allow us to establish compact expressions for the energy shifts that atomic transitions undergo.

Asymptotic formulas for the modified potential can be extracted from Eq. (35) without much efforts. For instance, at distances larger than the Compton wavelength $\lambda \sim m^{-1}$ of the axion, i.e., for $|\boldsymbol{x}| \gg \lambda$, the region $u \sim 1$ dominates in the integral involved in Eq. (35), and the axion-modified Coulomb potential approaches to

$$
\mathfrak{a}_{0}(|\boldsymbol{x}| \gg \lambda) \approx \frac{\mathfrak{q}}{4 \pi|\boldsymbol{x}|}\left[1+\frac{g^{2} m^{2}}{\pi^{2}} \frac{e^{-m|\boldsymbol{x}|}}{(m|\boldsymbol{x}|)^{4}}\right]
$$

However, at short distances $[|x| \ll \lambda]$, the main contribution to the integral in Eq. (35) results from the region $1 \leq u \leq(m|\boldsymbol{x}|)^{-1}$, and the integrand can be approached by its most slowly decreasing function in $u$, which is $\sim u e^{-m|x| u}$. Consequently,

$$
\mathfrak{a}_{0}(|\boldsymbol{x}| \ll \lambda) \approx \frac{\mathfrak{q}}{4 \pi|\boldsymbol{x}|}\left\{1+\frac{g^{2} m^{2}}{48 \pi^{2}} \frac{1}{(m|\boldsymbol{x}|)^{2}}\right\}
$$

This expression is independent of the axion mass. Observe that the distance $|\boldsymbol{x}|$ must satisfy the condition $|\boldsymbol{x}| \gg g /(4 \sqrt{3} \pi)$, otherwise our perturbative approach breaks down. Incidentally, the corresponding energy scale $\mu_{\mathrm{p}} \sim|\boldsymbol{x}|^{-1} \sim 4 \sqrt{3} \pi / g$ coincides—up to a numerical factor of the order of one-with the Landau pole linked to $\mathrm{QED}_{\mathrm{A}}$ : $\mu_{\mathrm{L}} \approx 4 \sqrt{6} \pi / g$ [for details see Ref. [66]].

The distortion of the Coulomb potential due to ALPs [see Eq. (35)] allows us to infer the induced modifications in the spectrum of a nonrelativistic hydrogenlike atom. Since so far no large deviations from the standard QED predictions have been observed, we will assume that these energy shifts are very small and, consequently, apply standard time-independent perturbation theory. When considering a first order approximation, the energy shift $\delta \varepsilon$ follows by averaging the correction to the electrostatic energy $\delta \mathscr{U}(\boldsymbol{x})=-e_{\mathrm{R}} \delta \mathfrak{a}(\boldsymbol{x})$ [see Eq. (33) and the Feynman diagram depicted in Fig. 4] over the 0th order wavefunctions $\left|\psi_{n, \ell, j}\right\rangle$. Explicitly,

$$
\delta \varepsilon_{n, \ell, j}^{(1)}=\left\langle\psi_{n, \ell, j}|\delta \mathscr{U}(\boldsymbol{x})| \psi_{n, \ell, j}\right\rangle .
$$

We wish to exploit this formula to predict a plausible axion Lamb-shift for the $2 s_{1 / 2}-2 p_{1 / 2}$ transition in atomic hydrogen. For this case, Eq. (38) leads to

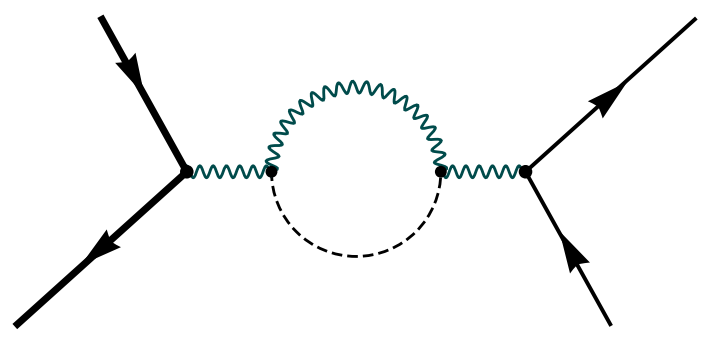

FIG. 4. Pictorial correction to the Coulomb potential due to quantum vacuum fluctuations of axion and electromagnetic fields. Leaving aside the electron (proton) legs [external lines with arrows in the right (left)], the remaining pieces of this diagram are described in Fig. 1.

$$
\begin{aligned}
\delta \varepsilon & =\delta \varepsilon_{2 s_{1 / 2}}^{(1)}-\delta \varepsilon_{2 p_{1 / 2}}^{(1)}, \\
& =\int_{0}^{\infty} d r r^{2} \delta \mathscr{U}(r)\left[R_{2 s}^{2}(r)-R_{2 p}^{2}(r)\right],
\end{aligned}
$$

where $r \equiv|\boldsymbol{x}|$ and $R_{n \ell}$ stands for a radial hydrogen wave function. In particular,

$$
\begin{aligned}
& R_{2 s}(r)=\frac{1}{\sqrt{2}} \frac{1}{a_{\mathrm{B}}^{3 / 2}}\left[1-\frac{r}{2 a_{\mathrm{B}}}\right] e^{-\frac{r}{2 a_{\mathrm{B}}}}, \\
& R_{2 p}(r)=\frac{1}{2 \sqrt{6}} \frac{r}{a_{\mathrm{B}}^{5 / 2}} e^{-\frac{r}{2 a_{\mathrm{B}}}} .
\end{aligned}
$$

Here $a_{\mathrm{B}}=\left(\alpha m_{e}\right)^{-1}$ is the Bohr radius with $\alpha=1 / 137$ the fine structure constant and $m_{e}=0.511 \mathrm{MeV}$ the electron mass.

Observe that the integration in Eq. (39) covers the region $[0, \infty)$. However, since $\mathrm{QED}_{\mathrm{A}}$ does not provide a precise information about the form of the axion-Coulomb potential for distances smaller than $g$, the integral over $r$ must be split $\int_{0}^{\infty} d r \ldots=\int_{0}^{g} d r \ldots+\int_{g}^{\infty} d r \ldots$. In the following we will assume that the contribution from the outer region $[g, \infty)$ dominates over the inner region $[0, g]$, which we ignore. ${ }^{5}$ As we will see very shortly, the yet undiscarded values for $g$ turn out to be much smaller than any characteristic atomic scale. With all these details in mind we integrate over $r$ and arrive at

$$
\delta \varepsilon \approx-\frac{\alpha g^{2}}{96 \pi^{2}} m^{4} a_{\mathrm{B}} \int_{1}^{\infty} \frac{d u}{u^{3}} \frac{\left[u^{2}-1\right]^{3}}{\left[1+u a_{\mathrm{B}} m\right]^{4}} e^{-g m u} .
$$

Let us study the asymptotes of this expression. We first consider the case in which $a_{\mathrm{B}} \gg \lambda$. Under this condition,

\footnotetext{
${ }^{5}$ Strictly speaking, in accordance with the treatment applied in Sec. II [read also below Eq. (35)], the splitting of the integral should be carried out at a certain point $d$ fulfilling the condition $d \gg g$. However, in order to avoid uncertainties stemming from this additional parameter, we set $d=g$. Our corresponding results should be considered as order-of-magnitude estimates, accordingly.
} 
the term of the integrand $\left[1+u a_{\mathrm{B}} m\right]^{4}$ is dominated by $u^{4} a_{\mathrm{B}}^{4} m^{4}$. The integral resulting from this approximation can be computed exactly. After a Taylor expansion in $m g \ll 1$, we find the compact expression

$$
\delta \varepsilon \approx \frac{\alpha g^{2}}{96 \pi^{2} a_{\mathrm{B}}^{3}}\left[\ln (g m)+\gamma+\frac{11}{12}\right] .
$$

As in Eq. (14), $\gamma=0.5772 \ldots$ refers to the Euler-Mascheroni constant. In the opposite case $a_{\mathrm{B}} \ll \lambda$, the integrand in Eq. (41) turns out to be a function that decreases monotonically with the growing of $u$. It is then justified to approach it through its most slowly decreasing part which is $\sim u^{3} e^{-m g u} /\left(1+u a_{\mathrm{B}} m\right)^{4}$. As a result, the corresponding integral can be computed analytically by using (3.353.1) in Ref. [87]. In the limit of $m g \ll 1$, it allows us to approach

$$
\delta \varepsilon \approx \frac{\alpha g^{2}}{96 \pi^{2} a_{\mathrm{B}}^{3}}\left[\ln \left(\frac{g}{a_{\mathrm{B}}}\right)+\gamma+\frac{11}{6}\right] .
$$

Notice that, the equation above is a good approximation whenever the condition $a_{\mathrm{B}} \gg g$ holds. Moreover, although Eqs. (41)-(43) apply for ordinary atomic hydrogen, they can be adapted conveniently for studying the same transition in other hydrogenlike atoms. When hydrogenlike ions with atomic number $Z>1$ are considered, for instance, the correction to the Lamb-shift will be given by Eqs. (41)(43), scaled by the factor $Z$ and $a_{\mathrm{B}} \rightarrow a_{\mathrm{B}} / Z$. If a muonic hydrogen atom is investigated instead, a replacement of the electron mass $m_{e}$ by the reduced mass of the system $m_{r} \approx$ $186 m_{e}$ would be required.

\section{Precision spectroscopy in $H_{\mu}$ and the proton radius anomaly}

Before continuing with the physics of virtual ALPs, we will estimate the contribution to the Lamb-shift by a meson whose interaction with the electromagnetic field resembles the one exhibited by ALPs [see Eq. (1)], i.e., the neutral pion $\pi^{0}$. We should however emphasize that its effect should be understood as a consequence of the quantum vacuum fluctuations of its constituent quark fields. When thinking of the axion as $\pi^{0}, m \rightarrow m_{\pi}=135 \mathrm{MeV}$ and the coupling constant $g \rightarrow \alpha /\left(\pi f_{\pi}\right)$ turns out to be determined by $\alpha$ and the pion decay constant $f_{\pi} \approx 92 \mathrm{MeV}$ [65]. Observe that the corresponding value of $g \approx 4.97 \times 10^{-3}$ fm is two orders of magnitude smaller than the proton radius $r_{\text {proton }} \approx 0.876 \mathrm{fm}$ [38]. The corresponding correction to the $2 s_{1 / 2}-2 p_{1 / 2}$ Lamb-shift in hydrogen atoms is $\delta \varepsilon=-1.07 \times 10^{-12} \mathrm{meV}$. This value turns out to be five orders of magnitude smaller than the experimental uncertainty $\left|\delta \varepsilon_{2 \sigma}\right|=2 \times 10^{-7} \mathrm{meV}$, established at $2 \sigma$ confidence level [35,37]. If the previous evaluation is carried out by considering a muonic hydrogen instead $\left[m_{e} \rightarrow m_{r}\right]$, we find that the correction to the energy due to the neutral pion field is $\delta \varepsilon=-6.81 \times 10^{-6} \mathrm{meV}$.
Since this is five orders of magnitude smaller than the existing discrepancy between the experimental measurement and the theoretical prediction $\delta \varepsilon=0.31 \mathrm{meV}$ $[38,42,45]$, virtual neutral pions are excluded as possible explanation for the proton radius puzzle.

Now, we wish to investigate whether the Lamb-shift induced by quantum vacuum fluctuations of axionlike fields might cure this anomaly. To this end, we will evaluate Eq. (41) considering a mass region in which reliable results can be extracted. Within a pure spectroscopy context, this occurs for ALP wavelengths smaller or of the order of the Bohr radius of $\mathrm{H}_{\mu}$, i.e., $\lambda \lesssim a_{\mu}$ with $a_{\mu} \approx 285 \mathrm{fm}$, otherwise interactions of other nature must be included. Correspondingly, we can formally explore ALP masses fulfilling the condition $1 \mathrm{MeV} \lesssim m$. However, in the range $1 \mathrm{MeV} \lesssim m \lesssim 100 \mathrm{MeV}$ the axion-diphoton coupling $g$ has been constrained severely from various results,

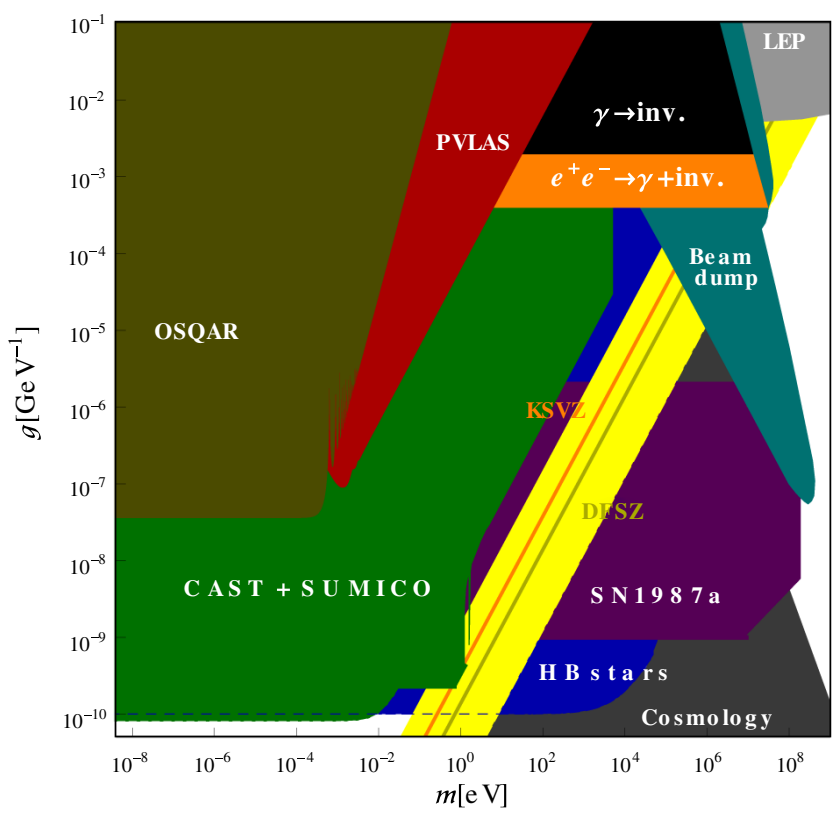

FIG. 5. Summary of exclusion areas for a pseudoscalar ALP coupled to two photons. Compilation adapted from Refs. [15-19]. The picture includes [inclined yellow band] the predictions of the axion models $|E / N-1.95|=0.07-7$ (the notation of this formula is in accordance with Ref. [90]). Colored in orange and black appear the regions ruled out by particle decay experiments. While the portion discarded by investigating the energy loss in the horizontal branch (HB) stars are shown in blue, the excluded area resulting from the solar monitoring of a plausible ALP flux $(\mathrm{CAST}+\mathrm{SUMICO})$ has been added in green. In purple the portion discarded by measuring the duration of the neutrino signal of the supernova SN1987A is depicted, whereas the dark gray area results from cosmological studies. The excluded area in dark turquoise has been established from beam dump experiments. Besides, the light gray zone has been excluded from electronpositron collider (LEP) investigations. Finally, the colored sectors in olive and red show the exclusion regions corresponding to OSCAR and PVLAS collaborations, respectively. 

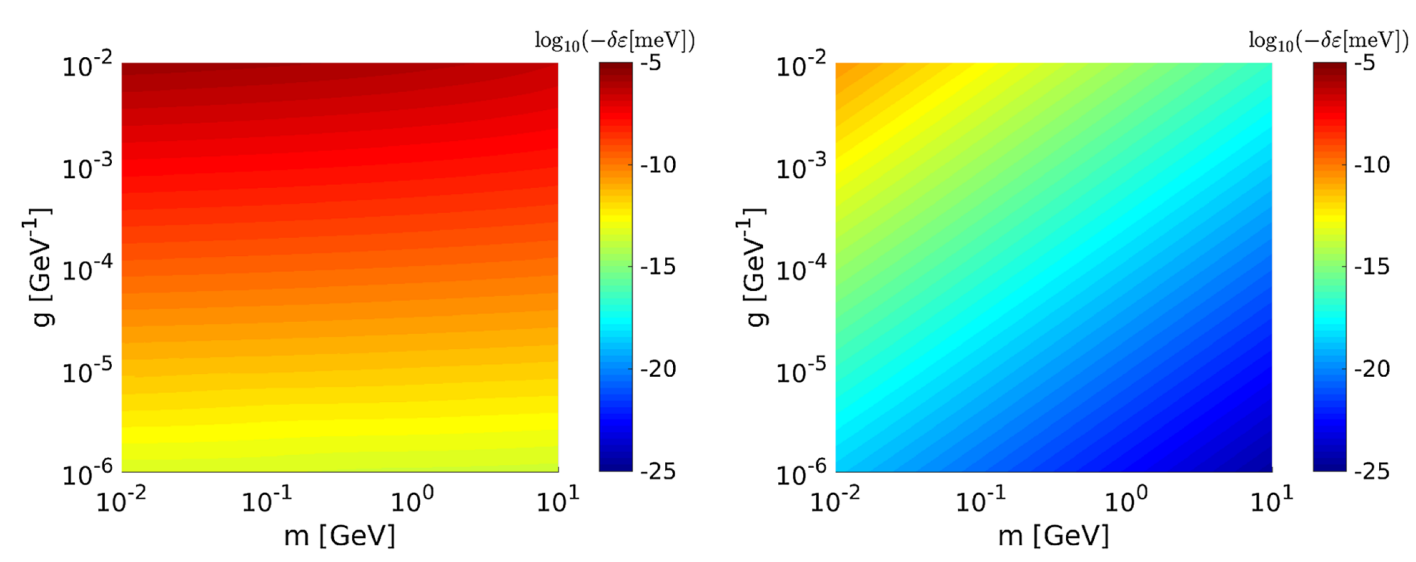

FIG. 6. Energy-shift induced by hypothetical quantum vacuum fluctuations of axionlike fields on the $2 s_{1 / 2}-2 p_{1 / 2}$ (left panel) and the $3 p_{1 / 2}-3 d_{1 / 2}$ (right panel) atomic transitions in $\mathrm{H}_{\mu}$. The ALPs parameters used in these numerical evaluations are still undiscarded. While the results exhibited in the left panel rely on Eq. (41), the outcomes depicted in the right panel follow from Eq. (45). Observe that the energy shift is plotted in the form of $\log _{10}(-\delta \varepsilon[\mathrm{meV}])$.

including those dealing with electron beam fixed-target setups [see compilation of bounds in Refs. [88,89]]. Conversely, the sensitivities in experiments where ALPs masses $100 \mathrm{MeV} \lesssim m \lesssim 10 \mathrm{GeV}$ are probed turn out to be much weaker [white sector in the right hand side of Fig. 5]. A recent investigation based on electron-positron colliders has constrained $g$ to lie below $g<10^{-2} \mathrm{GeV}^{-1}$ [89].

A numerical assessment of the axion-modified Lambshift has been carried out by considering this yet undiscarded region. The outcome of this evaluation is summarized in the left panel of Fig. 6. Observe that the energy shift has been plotted in the form of $\log _{10}(-\delta \varepsilon[\mathrm{meV}])$. The highest value achieved for $\delta \varepsilon \sim-10^{-6} \mathrm{meV}$ corresponds to $g \sim$ $10^{-2} \mathrm{GeV}^{-1}$ and $m \sim 10^{-2} \mathrm{GeV}$. Toward higher axion masses $m \sim 10 \mathrm{GeV}$ and lower axion-diphoton couplings $g \sim 10^{-6} \mathrm{GeV}^{-1}$ the correction to the Lamb-shift tends to decrease significantly $\left[\delta \varepsilon \sim-10^{-14} \mathrm{meV}\right]$. Both estimates coincide with the values resulting from Eq. (42). The smallness of these outcomes as compared with the aforementioned discrepancy rules out the corresponding virtual ALPs as candidates to explain the $\mathrm{H}_{\mu}$ anomaly. As we have anticipated above Eq. (41), the chosen values for $10^{-7} \mathrm{fm} \lesssim$ $g \lesssim 10^{-4} \mathrm{fm}$ are much smaller than $r_{\text {proton }} \approx 0.876 \mathrm{fm}$.

Clearly, the previous statements cannot be considered conclusive as our estimation undergoes theoretical uncertainties arising from both the internal limitation of $\mathrm{QED}_{\mathrm{A}}$ at short distances as well as the finite proton size. The latter being closely related to the fact that the $s$-states penetrate the nucleus deeply even for the chosen $g$. This last problem can be relaxed if transitions between excited states with nonzero angular momentum are considered instead. Although the problem of their relatively short lifetimes constitutes a major issue for their experimental investigation, their measurements seem to be a priori a reliable way to have a cleaner picture of whether a certain ALP is the cause of the aforementioned discrepancy or not. Inspired by these arguments, we consider as an example the $3 p_{1 / 2}-3 d_{1 / 2}$ transition. In this case, the required wave functions are

$$
\begin{aligned}
& R_{3 p}(r)=\frac{4 \sqrt{2}}{9} \frac{1}{\left(3 a_{\mathrm{B}}\right)^{3 / 2}}\left(\frac{r}{a_{\mathrm{B}}}\right)\left[1-\frac{r}{6 a_{\mathrm{B}}}\right] \mathrm{e}^{-\frac{r}{3 a_{\mathrm{B}}}}, \\
& R_{3 d}(r)=\frac{2 \sqrt{2}}{27 \sqrt{5}} \frac{1}{\left(3 a_{\mathrm{B}}\right)^{3 / 2}}\left(\frac{r}{a_{\mathrm{B}}}\right)^{2} \mathrm{e}^{-\frac{r}{3 a_{\mathrm{B}}}}
\end{aligned}
$$

An adequate replacement of the radial wave functions in Eq. (39) by those above allows us to determine the corresponding modification of the transition energy:

$$
\begin{aligned}
\delta \varepsilon \approx & -\frac{\alpha g^{2} m^{2}}{108 \pi^{2} a_{\mathrm{B}}} \int_{1}^{\infty} \frac{d u}{u^{5}} \frac{\left[u^{2}-1\right]^{3}}{\left[1+\frac{3}{2} u a_{\mathrm{B}} m\right]^{4}} \\
& \times\left\{1-\frac{2}{1+\frac{3}{2} u a_{\mathrm{B}} m}+\frac{1}{\left[1+\frac{3}{2} u a_{\mathrm{B}} m\right]^{2}}\right\} .
\end{aligned}
$$

In the limit $a_{\mathrm{B}} \gg \lambda$, the expression above is well approached by $\delta \varepsilon \approx-\alpha g^{2} /\left(4374 \pi^{2} m^{2} a_{\mathrm{B}}^{5}\right)$. The expressions associated with $\mathrm{H}_{\mu}$ can be read off from the previous one by replacing $a_{\mathrm{B}} \rightarrow a_{\mathrm{B}} /(186)$. Taking as a reference the undiscarded region used previously, $100 \mathrm{MeV} \lesssim m \lesssim 10 \mathrm{GeV}$ with $g<10^{-2} \mathrm{GeV}^{-1}$, Eq. (45) has been evaluated. The result of this assessment is shown in the right panel of Fig. 6. As in the $2 s_{1 / 2}-2 p_{1 / 2}$ transition, the highest energy-shift linked to the $3 p_{1 / 2}-3 d_{1 / 2}$ transition in $\mathrm{H}_{\mu}$ arises from the combination of $g \sim 10^{-2} \mathrm{GeV}^{-1}$ and $m=10^{-2} \mathrm{GeV}$. In such a case $\delta \varepsilon \sim-10^{-11} \mathrm{meV}$, which is five orders of magnitude smaller than the outcome associated with the axion $2 s_{1 / 2}-2 p_{1 / 2}$ Lamb-shift. It is worth emphasizing that, while the uncertainty introduced by the use of spherically symmetric orbitals could be circumvented as described above, the one linked to the physics at distances shorter than $g$ remains. 

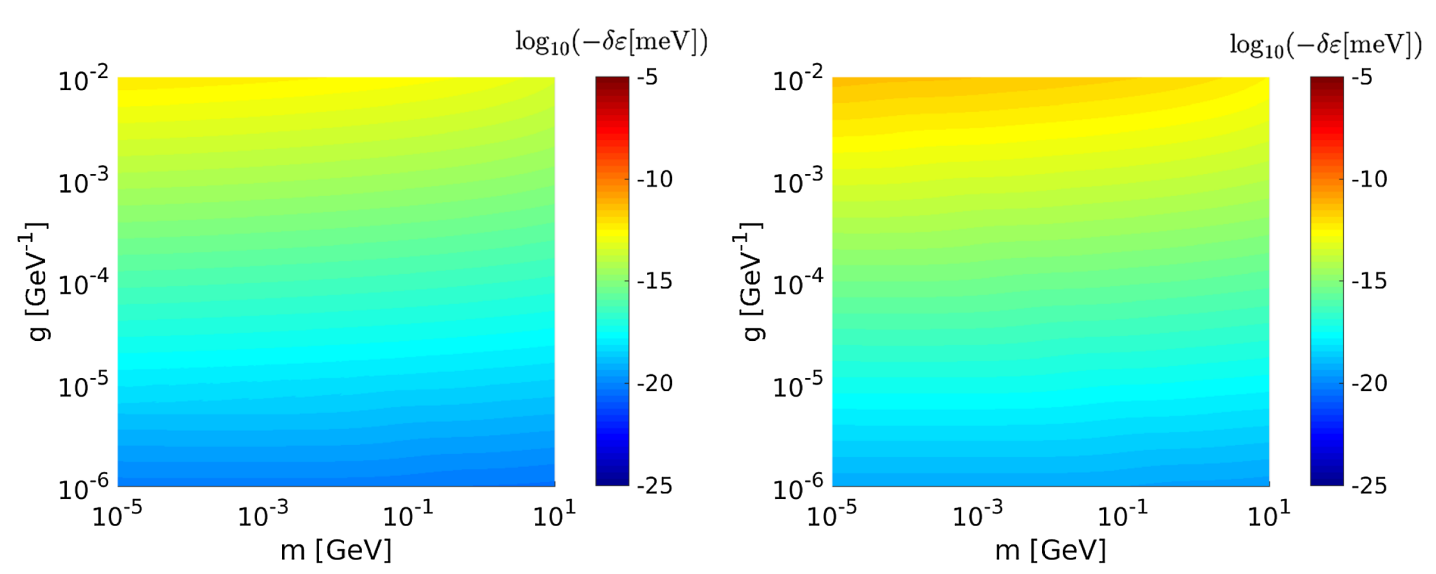

FIG. 7. Projected sensitivities for the $2 s_{1 / 2}-2 p_{1 / 2}$ [see Eq. (41)] and $1 s_{1 / 2}-2 s_{1 / 2}$ [see Eq. (46)] atomic transitions in hydrogen are depicted in the left and the right panel, respectively. In contrast to Fig. 6, the region for the axion mass $m$ is wider, covering from $10^{-5} \mathrm{GeV}$ to $10 \mathrm{GeV}$. As previously, $10^{-6} \mathrm{GeV}^{-1}<g<10^{2} \mathrm{GeV}^{-1}$ and the energy-shift is given in the form of $\log _{10}(-\delta \varepsilon[\mathrm{meV}]$.

\section{Sensitivity to ALPs in high-precision hydrogen spectroscopy}

In this section we want to investigate whether the current sensitivity in atomic hydrogen can improve the existing bounds on the axion parameter space. To do this we will analyse the respective energy-shift in both $2 s_{1 / 2}-$ $2 p_{1 / 2}$ and $1 s_{1 / 2}-2 s_{1 / 2}$ transitions. An expression for the latter $\delta \varepsilon=\int_{0}^{\infty} d r r^{2} \delta \mathscr{U}(r)\left[R_{1 s}^{2}(r)-R_{2 s}^{2}(r)\right]$ can be easily determined by taking into account the formula for $R_{2 s}(r)$ in Eq. (40) and the radial part of the $1 s_{1 / 2}$-state: $R_{1 s}(r)=2 \exp \left[-r / a_{\mathrm{B}}\right] / a_{\mathrm{B}}^{3 / 2}$. Explicitly,

$$
\begin{aligned}
\delta \varepsilon= & -\frac{\alpha g^{2} m^{2}}{48 \pi^{2} a_{\mathrm{B}}} \int_{1}^{\infty} \frac{d u}{u^{5}}\left[u^{2}-1\right]^{3} e^{-g m u}\left\{\frac{1}{\left[1+\frac{1}{2} u a_{\mathrm{B}} m\right]^{2}}\right. \\
& \left.-\frac{1}{2} \frac{1}{\left[1+u a_{\mathrm{B}} m\right]^{2}}\left(1-\frac{2}{1+u a_{\mathrm{B}} m}+\frac{3}{2\left[1+u a_{\mathrm{B}} m\right]^{2}}\right)\right\} .
\end{aligned}
$$

While the left panel in Fig. 7 shows the energy shift for $2 s_{1 / 2}-2 p_{1 / 2}$ [see Eq. (41)], the one in the right depicts the result associated with the $1 s_{1 / 2}-2 s_{1 / 2}$ transition [see Eq. (46)]. Both evaluations have been carried out by considering the region of the coupling $10^{-6} \mathrm{GeV}^{-1}<g<$ $10^{-2} \mathrm{GeV}^{-1}$. In contrast to $\mathrm{H}_{\mu}$, reliable predictions from high-precision spectroscopy in ordinary hydrogen require to deal with ALPs masses $m \gtrsim 10^{-5} \mathrm{GeV}$, corresponding to wavelengths $\lambda \lesssim a_{\mathrm{B}}$. The highest mass shown in both panels $[m=10 \mathrm{GeV}]$ has been set in order to preserve the perturbative condition $m g \ll 1$.

When comparing the energy shifts resulting from each panel in Fig. 7 with the corresponding experimental uncertanities $\left(\left|\delta \varepsilon_{2 \sigma}\right|=2 \times 10^{-7} \mathrm{meV}\right.$ for $2 s_{1 / 2}-2 p_{1 / 2}$ and $\left|\delta \varepsilon_{1 \sigma}\right|=1 \times 10^{-6} \mathrm{meV}$ for $1 s_{1 / 2}-2 s_{1 / 2}$ transition [37]) we conclude that, in order to improve the current bounds on the axion parameter space, an enhancement in sensitivity of at least five orders of magnitude is required. It is worth remarking that this sensitivity gap also manifests in other high-precision experiments searching for potential deviations of the Coulomb's law as those of Cavendishtype. For further details we refer the reader to Appendix B. This lack of sufficient sensitivity in the context of ALPs is significant when taking into account that these setups have allowed for constraining severely the parameter spaces of other weakly interacting sub-eV particles, including paraphotons and minicharged particles. However, we should emphazise that-in contrast to our investigation-these particle candidates have been treated within renormalizable frameworks and, thus, the bounds have been established on dimensionless coupling constants. Likewise, we have already indicated below Eq. (16) that in the axion theory the quantity playing the corresponding role combines two unknown parameters $\sim g m$. Hence, the axion mass $m$ suppresses the limits that can be inferred for $g$.

\section{CONCLUSION}

Within the effective framework of axion quantum electrodynamics, terms beyond the minimal coupling of two photons to a neutral pseudoscalar field have been used to renormalize the polarization tensor and the axion selfenergy operator. The former outcome was used to establish the photon propagator distorted by the quantum vacuum fluctuations of axionlike fields, a piece essential for determining the modification of the Coulomb potential induced by both virtual photons and ALPs. This result allowed us to evaluate the way in which atomic spectra could change. Particular attention has been paid to the $2 s_{1 / 2}-2 p_{1 / 2}$ transition in hydrogenlike atoms as it might constitute the most natural way of verifying our predictions experimentally. Likewise, this sort of axionmodified Lamb-shift has been considered in attempting 
to explain the proton radius anomaly in muonic hydrogen. By contrasting the experimental result with our theoretical prediction, it was found that - up to the uncertainties caused by the nature of the transition and the internal limitations of axion-electrodynamics-ALPs can be excluded as plausible candidates for solving the aforementioned problem.

Our investigation has revealed explicitly that neither atomic spectroscopy nor experiments of Cavendish-type allow us to infer bounds that improve the existing constraints on the axion parameter space. This fact contrasts with analogous outcomes linked to scenarios containing minicharged particles and hidden photon fields, in which both precision techniques have turned out to be particularly valuable [34-37]. The loss of sensitivity within the axion context is conceptually rooted in the nonrenormalizable character of $\mathrm{QED}_{\mathrm{A}}$ and manifests - at the level of the modified Coulomb potential Eq. (35) - through the dimensionless factor $\sim g m$. This ratio of scales accomplishing somewhat a role similar to the coupling strengths of the photon-paraphoton mixing $\chi$ and the parameter $\epsilon$ in the minicharged particles scenario. To a certain extent the described problem justifies the existing demand for new laboratory-based routes looking for ALPs $[13,14]$ by using strong electromagnetic fields [15-18], e.g., those offered by high-intensity lasers [91-101].

Let us finally remark that the expression for $\Pi_{\mathrm{R}}^{\mu \nu}\left(p_{1}, p_{2}\right)$ [see Eq. (28)] constitutes an essential piece for a more general class of polarization tensors which result when external electromagnetic fields polarize the vacuum $[80,91]$.

\section{ACKNOWLEDGMENTS}

The authors thank A. B. Voitkiv and A. E. Shabad for useful discussions. S. V.-C. and C. M. gratefully acknowledge funding by the German Research Foundation (DFG) under Grant No. MU 3149/5-1.

\section{APPENDIX A: PARTICLE-GHOST CONTENT OF THE GAUGE SECTOR AND AN ALTERNATIVE FOUR-FIELDS FORMULATION OF AXION-ELECTRODYNAMICS}

As mentioned in Sec. II A, the photon sector also contains Pauli-Villars ghosts. In order to show this, let us consider the corresponding Green function resulting from a covariant quantization of $\bar{a}^{\mu}(x)$ via a path integral representation. When fixing the gauge via $\mathcal{L}_{\text {gauge }}=-\frac{1}{2}\left[\left(1+\bar{g}^{2} \overline{\mathfrak{b}}_{a}^{2} \square\right) \partial_{\mu} \bar{a}^{\mu}\right]^{2}$ it turns out to be $[102,103]$ :

$$
G_{\alpha \beta}\left(p^{2}\right)=\left[-\frac{1}{p^{2}}+\frac{1}{p^{2}-\bar{m}_{\mathrm{gh}}^{2}}\right] \mathscr{g}_{\alpha \beta},
$$

where $\bar{m}_{\text {gh }}^{2}=\left(\bar{g}_{\mathfrak{b}_{a}}\right)^{-2}$ is the corresponding bare ghost mass. Here, a longitudinal contribution $\sim p_{\alpha} p_{\beta}$ has been ignored on the grounds that, if the photons couple to a conserved current $j^{\mu}(x)$, i.e., $p_{\mu} j^{\mu}(p)=0$, a term of this nature does not contribute to the S-matrix elements. Manifestly, the photon Green function in Eq. (A1) resembles Eq. (3). However, the particle-ghost content linked to this expression is somewhat blurred owing to the presence of the metric tensor $q_{\alpha \beta}$. To highlight the emergence of the Pauli-Villars ghost—leaving aside those unphysical states linked to the quantization procedure that eventually must cancel each other-we will follow a method that has been used previously within the context of quantum gravity $[68,104,105] .^{6}$ Rather than dealing with the expression above directly, one introduces the saturated Green function $\mathscr{G}\left(p^{2}\right)=j^{\alpha} G_{\alpha \beta}\left(p^{2}\right) j^{\beta}$ and investigates its residues at each pole: $p^{2}=0$ and $p^{2}=\bar{m}_{\mathrm{gh}}^{2}$. As for any $m \geq 0$ the relation $\left.j^{2}\right|_{p^{2}=m^{2}}<0$ holds-see proof of Lemma 1 in Ref. [68] - a physical particle is linked to a nonnegative residue of $\mathscr{G}\left(p^{2}\right)$, whereas a ghost emerges when the contrary occurs. For the case under consideration then follows that $\left.\operatorname{Res} \mathscr{G}\left(p^{2}\right)\right|_{p^{2}=0}>0$ (photon) and $\left.\operatorname{Res} \mathscr{G}\left(p^{2}\right)\right|_{p^{2}=\bar{m}_{\mathrm{gh}}^{2}}<0$ (ghost).

Noteworthy, the decompositions of the axion and photon Green functions [see Eqs. (3) and (A1)] suggest that the effects of the higher-dimensional operators can be formulated in terms of auxiliary-fictitious-fields. In this context, the action of interest reads

$$
\begin{aligned}
\mathcal{S}_{\text {eff }}= & \int d^{4} x\left\{-\frac{1}{4} f_{\mu \nu} f^{\mu \nu}+\frac{1}{2} \partial_{\mu} \bar{\phi} \partial^{\mu} \bar{\phi}-\frac{1}{2} \bar{m}^{2} \bar{\phi}^{2}-\Phi \square \bar{\phi}\right. \\
& \left.+\frac{1}{2} \bar{m}_{\mathrm{s}}^{2} \Phi^{2}-\frac{1}{2} \bar{m}_{\mathrm{gh}}^{2} \mathscr{A}^{2}+\frac{1}{2} f_{\mu \nu} \mathscr{F}^{\mu \nu}+\frac{1}{4} \bar{g} \bar{\phi} \tilde{f}_{\mu \nu} f^{\mu \nu}\right\},
\end{aligned}
$$

where $\mathscr{F}_{\mu \nu}=\partial_{\mu} \mathscr{A}_{\nu}-\partial_{\nu} \mathscr{A}_{\mu}$ and $\bar{m}_{\mathrm{s}}^{2}=\left(\bar{g} \overline{\mathfrak{b}}_{\phi}\right)^{-2}$. We remark that the equations of motion for the auxiliary fields are exact

$$
\Phi=\frac{1}{\bar{m}_{\mathrm{s}}^{2}} \square \bar{\phi}, \quad \mathscr{A}_{\lambda}=-\frac{1}{\bar{m}_{\mathrm{gh}}^{2}} \partial_{\mu} f_{\lambda}^{\mu}
$$

Hence, when integrating out both $\Phi(x)$ and $\mathscr{A}_{\lambda}(x)$ classically, i.e., by removing them from $\mathcal{S}_{\text {eff }}$ using their equations of motion, we reproduce the action of axion-electrodynamics extended by terms proportional to $\bar{g}^{2}$, i.e., $\mathcal{S}_{\text {eff }} \rightarrow \mathcal{S}=S_{\bar{g}}+S_{\bar{g}^{2}}$ [see Eqs. (1) and (2)].

Observe that, as a consequence of the shift $\bar{a} \rightarrow a+\mathscr{A}$ and $\bar{\phi} \rightarrow \phi-\Phi$, the functional action in Eq. (A2) can be written as

\footnotetext{
${ }^{6}$ We precise that higher-derivate operators in combination with nonlocal terms emerge in many other interesting theoretical scenarios, e.g., in construction of effective quantum field theories accounting for quantum conformal and chiral anomalies (see e.g., [106] and references therein).
} 


$$
\begin{aligned}
\mathcal{S}_{\mathrm{eff}}= & \int d^{4} x\left\{-\frac{1}{4} f_{\mu \nu} f^{\mu \nu}+\frac{1}{4} \mathscr{F}_{\mu \nu} \mathscr{F}^{\mu \nu}-\frac{1}{2} \bar{m}_{\mathrm{gh}}^{2} \mathscr{A}^{2}\right. \\
& +\frac{1}{2} \partial_{\mu} \phi \partial^{\mu} \phi-\frac{1}{2} \bar{m}^{2}(\phi-\Phi)^{2}-\frac{1}{2} \partial_{\mu} \Phi \partial^{\mu} \Phi+\frac{1}{2} \bar{m}_{\mathrm{s}}^{2} \Phi^{2} \\
& \left.+\frac{1}{4} \bar{g}(\phi-\Phi)\left[\tilde{f}_{\mu \nu} f^{\mu \nu}+2 \tilde{\mathscr{F}}_{\mu \nu} f^{\mu \nu}+\tilde{\mathscr{F}}_{\mu \nu} \mathscr{F}^{\mu \nu}\right]\right\} .
\end{aligned}
$$

Clearly, the first term contained in this formula is the Maxwell Lagrangian, while the combination of the two remaining contributions in the first line somewhat looks like the Proca Lagrangian, with the exception that the sign of its kinetic term is not the usual one. Likewise, the kinetic portion linked to the $\Phi(x)$ field manifests an opposite sign to the corresponding contribution of $\phi(x)$. Owing to the described feature the associated Hamiltonian is not positive defined leading - upon quantization - to the absence of a ground state. The particle-ghost content of the theory is elucidated in this alternative formulation to the requirement that free fields of particles (ghosts) have positive (negative) energy.

\section{APPENDIX B: SENSITIVITY TO ALPS IN EXPERIMENTS OF CAVENDISH-TYPE}

Precision tests of Coulomb's law via Cavendish-type experiments have severely constricted the parameter space of hidden photons in the $\mu \mathrm{eV}$ mass regime [34,107]. Today, these setups also provide the best laboratory bounds on mini-charged particles in the sub- $\mu \mathrm{eV}$ range [36]. Here, we want to estimate the sensitivity of this type of experiments in the context of ALPs. To this end, we consider a setup containing two concentric spheres: an outer charged conducting sphere-characterized by a radius $b$-and an uncharged conducting inner sphere with a radius $a$. Only if the electrostatic potential follows the $r^{-1}$ law, the potential difference between the spheres vanishes and the cavity is free of electromagnetic field. However, deviations from the Coulomb potential like those induced by loop corrections [compare Eq. (35)] could lead to a nontrivial relative voltage difference $\gamma_{a b}=|[\mathfrak{u}(\mathbb{Q}, b, b)-\mathfrak{u}(\mathbb{Q}, a, b)] / \mathfrak{u t}(\mathbb{Q}, b, b)|$ that can be detected. This observable depends on the potential of the charged sphere evaluated on its surface $\mathfrak{t}(\mathbb{Q}, b, b)$ as well as on the surface of the inner sphere $\mathfrak{t}(\mathscr{Q}, a, b)$.

In general, the electrostatic potential of a sphere with radius $b$ and charge $\mathbb{Q}$ evaluated at a distance $r$ from its center has the form

$$
\begin{aligned}
\mathfrak{t}(\mathbb{Q}, r, b) & =\frac{\mathbb{Q}}{2 b r}[f(r+b)-f(|r-b|)], \\
f(r) & =\int_{0}^{r} s \mathscr{A}_{0}(s) d s,
\end{aligned}
$$

where $\mathscr{A}_{0}(s)$ is an arbitrary potential in which the charge of the pointlike particle must be set to unity [108]. Now, to determine $\gamma_{a b}$ resulting from $\mathrm{QED}_{\mathrm{A}}$ we insert the axion- modified Coulomb potential $\mathfrak{a}_{0}(s, \mathfrak{q}=1)$ [see Eq. (35)] into the expression above. Notice that, similarly to the case analyzed in Sec. III B, the integral over $s$ must be split into two parts: $\int_{0}^{r} d s \ldots=\int_{0}^{g} d s \ldots+\int_{g}^{r} d s \ldots$ Ignoring the contribution coming from the region $[0, g]$ we obtain

$$
\begin{aligned}
\gamma_{a b} \approx & \mid \frac{g^{2} m}{96 \pi^{2}} \int_{1}^{\infty} \frac{d u}{u^{6}}\left[u^{2}-1\right]^{3}\left\{\frac{e^{-2 b m u}-e^{-g m u}}{b}\right. \\
& \left.+\frac{2}{a} e^{-b m u} \sinh (a m u)\right\}+\mathcal{O}\left(g^{4} m^{2}\right) \mid
\end{aligned}
$$

The integral that remains in this formula can be calculated analytically by using (3.351.4) in Ref. [87]. Since both $b$ and $a$ are macroscopic quantities, the conditions $a, b, b-$ $a \gg g$ hold and we can approximate the expression above by

$$
\gamma_{a b} \approx\left|\frac{g^{2} m}{96 \pi^{2} b} \int_{1}^{\infty} \frac{d u}{u^{6}}\left[u^{2}-1\right]^{3} e^{-g m u}\right| .
$$

Notice that Eq. (B3) is independent of the radius $a$ of the inner sphere. When considering the limit $m g \ll 1$ we obtain

$$
\gamma_{a b} \approx \frac{g}{96 \pi^{2} b},
$$

which does not depend on the axion mass $m$ either.

With $\gamma_{a b}$ to our disposal, we can proceed to estimate the sensitivity of this setup in the search for ALPs. For such a purpose, we use the benchmark parameters of the experiment performed by Plimpton and Lawton $[a=38 \mathrm{~cm}$, $b=46 \mathrm{~cm}]$ in which a margin for $\gamma_{a b}$ exists, provided it lies below $\left|\gamma_{a b}\right| \lesssim 3 \times 10^{-10}$ [109]. By making use of Eq. (B4) we find

$$
g \lesssim 6.7 \times 10^{7} \mathrm{GeV}^{-1}
$$

We emphasize that - as a consequence of the perturbative condition [see below Eq. (B3)] - this result applies for $m \ll 15 \mathrm{eV}$. Besides, it is trustworthy for axion wavelengths smaller than the typical length scale of the spheres $\sim 0.1 \mathrm{~m}$, i.e., for axion masses $m \gg 10^{-6} \mathrm{eV}$. We note that the constraint in Eq. (B5) for the mass region $10^{-6} \mathrm{eV} \ll$ $m \ll 15 \mathrm{eV}$ has already been discarded by combining the experimental outcomes of collaborations such as PVLAS and OSCAR [see Fig. 5]. Hence, the sensitivity in this experiment of Cavendish-type is not high enough to improve the existing bounds on the axion parameter space.

It is worth remarking that a more accurate version of this kind of experiments has been carried out by using four concentric icosahedrons [110]. For obtaining first estimates, they may be treated approximately as four concentric spheres. In contrast to the setup of Plimpton and 
Lawton, here a very high voltage is applied between the outer two spheres with radii $d=127 \mathrm{~cm}$ and $c=94.7 \mathrm{~cm}$. The voltage difference is measured between the two internal ones, with radii $b=94 \mathrm{~cm}$ and $a=60 \mathrm{~cm}$, which are uncharged. This setup allows us to infer bounds for the ALPs parameters via the ratio between the voltage differences:

$\gamma_{a b c d}=\left|\frac{\mathfrak{u}(\mathscr{Q}, c, b)-\mathfrak{u}(\mathscr{Q}, d, b)-\mathfrak{u}(\mathscr{Q}, c, a)+\mathfrak{u}(\mathscr{Q}, d, a)}{2 \mathfrak{u}(\mathbb{Q}, c, d)-\mathfrak{u}(\mathscr{Q}, d, d)-\mathfrak{u}(\mathbb{Q}, c, c)}\right|$.

We insert Eq. (35) into (B1) and evaluate the resulting formula in the various parameters contained in Eq. (B6). As a consequence, we end up with

$$
\begin{aligned}
\gamma_{a b c d}= & \mid \frac{g^{2} m c}{48 \pi^{2} \delta} \int_{1}^{\infty} d u\left[1-\frac{1}{u^{2}}\right]^{3} e^{-m d u} \\
& \times\left[1-\frac{d}{c} e^{-m \delta u}\right]\left[\frac{\sinh (m b u)}{b}-\frac{\sinh (m a u)}{a}\right] \mid,
\end{aligned}
$$

where $\delta \equiv c-d$ and terms of the order of $\sim g^{4} m^{2}$ have been disregarded. Notice that, in contrast to Eq. (B2), the integrand above lacks terms involving $\sim e^{-m g u}$. This could be anticipated because the numerator of $\gamma_{a b c d}$ [see Eq. (B6)] does not contain a potential evaluated at the surface of the spheres [compare with $\gamma_{a b}$ given above Eq. (B1)].
Consequently, when the condition $m d=d / \lambda \ll 1$ is satisfied, the asymptotic expression for $\gamma_{a b c d}$ becomes independent of the axion mass and quadratic in $g$ :

$$
\begin{aligned}
\gamma_{a b c d} \approx & \mid \frac{g^{2}}{96 \pi^{2}} \frac{c}{b(c-d)(d-b)} \\
& \times\left(1-\frac{b(d-b)}{a(d-a)}-\frac{d(d-b)}{c(c-b)}+\frac{d b(d-b)}{a c(c-a)}\right) \mid .
\end{aligned}
$$

Since this formula applies for axion wavelengths larger than the typical length scale of the experiment, the outcomes resulting from it can be considered reliable as long as the interactions between ALPs and plausible fields/ matter existing outside of the external icosahedron are negligible. Next, the aforementioned experiment achieves a precision $\left|\gamma_{a b c d}\right| \lesssim 2 \times 10^{-16}[36,110]$. Combining this value with Eq. (B8) we constraint $g$ to lie below

$$
g \lesssim 9.8 \times 10^{7} \mathrm{GeV}^{-1} \text { for } m \ll 10^{-7} \mathrm{eV} .
$$

Noteworthy, despite the improvement in the experimental accuracy, the resulting upper limit turns out to be comparable to the one found from the results of Plimpton and Lawton [see Eq. (B5)] and so, no improvement is found as compared with the existing constraints. The lack of sensitivity is understood here as a direct consequence of the quadratic dependence of $\gamma_{a b c d}$ on $g$ [see Eq. (B8)].
[1] R. D. Peccei and H. R. Quinn, $C P$ Conservation in the Presence of Pseudoparticles, Phys. Rev. Lett. 38, 1440 (1977).

[2] F. Wilczek, Problem of Strong P and T Invariance in the Presence of Instantons, Phys. Rev. Lett. 40, 279 (1978).

[3] S. Weinberg, A New Light Boson?, Phys. Rev. Lett. 40, 223 (1978).

[4] L. Covi, J. E. Kim, and L. Roszkowski, Axinos as Cold Dark Matter, Phys. Rev. Lett. 82, 4180 (1999).

[5] G. G. Raffelt, Axions-motivation, limits and searches, J. Phys. A 40, 6607 (2007).

[6] L. D. Duffy and K. van Bibber, Axions as dark matter particles, New J. Phys. 11, 105008 (2009).

[7] P. Sikivie, Dark matter axions, Int. J. Mod. Phys. A 25, 554 (2010).

[8] H. Baer, A. D. Box, and H. Summy, Neutralino versus axion/axino cold dark matter in the 19 parameter SUGRA model, J. High Energy Phys. 10 (2010) 023.

[9] E. Witten, Some properties of $\mathrm{O}(32)$ superstrings, Phys. Lett. 149B, 351 (1984).

[10] P. Svrcek and E. Witten, Axions in string theory, J. High Energy Phys. 06, (2006) 051.
[11] O. Lebedev and S. Ramos Sanchez, The NMSSM and string theory, Phys. Lett. B 684, 48 (2010).

[12] M. Cicoli, M. Goodsell, and A. Ringwald, The type IIB string axiverse and its low-energy phenomenology, J. High Energy Phys. 10 (2012) 146.

[13] J. Jaeckel, E. Masso, J. Redondo, A. Ringwald, and F. Takahashi, We need lab experiments to look for axion-like particles, arXiv:hep-ph/0605313.

[14] J. Jaeckel, E. Massó, J. Redondo, A. Ringwald, and F. Takahashi, Need for purely laboratory-based axionlike particle searches, Phys. Rev. D 75, 013004 (2007).

[15] J. Jaeckel and A. Ringwald, The low-energy frontier of particle physics, Annu. Rev. Nucl. Part. Sci. 60, 405 (2010).

[16] A. Ringwald, Exploring the role of axions and other WISPs in the dark Universe, Phys. Dark Universe 1, 116 (2012).

[17] J. L. Hewett et al., in Fundamental physics at the intensity frontier, The Proceedings of the 2011 workshop on Fundamental Physics at the Intensity Frontier (Argone National Laboratory, 2012), DOI: 10.2172/1042577.

[18] R. Essig et al., Working group report: New light weakly coupled particles, arXiv:1311.0029. 
[19] S. Alekhin et al., A facility to search for hidden particles at the CERN SPS: The SHiP physics case, Rep. Prog. Phys. 79, 124201 (2016).

[20] F. Wilczek, Two Applications of Axion Electrodynamics, Phys. Rev. Lett. 58, 1799 (1987).

[21] A. S. Chou, W. Wester, A. Baumbaugh, H. R. Gustafson, Y. Irizarry-Valle, P. O. Mazur, J. H. Steffen, R. Tomlin, X. Yang, and J. Yoo (GammeV (T-969) Collaboration), Search for Axion-Like Particles Using a Variable Baseline Photon Regeneration Technique, Phys. Rev. Lett. 100, 080402 (2008).

[22] J.H. Steffen and A. Upadhye, The GammeV suite of experimental searches for axion-like particles, Mod. Phys. Lett. A 24, 2053 (2009).

[23] A. Afanasev, O. K. Baker, K. B. Beard, G. Biallas, J. Boyce, M. Minarni, R. Ramdon, M. Shinn, and P. Slocum, New Experimental Limit on Optical Photon Coupling to Light Neutral, Scalar Bosons, Phys. Rev. Lett. 101, 120401 (2008).

[24] P. Pugnat (OSQAR Collaboration), Results from the OSQAR photon regeneration experiment: No light shining through a wall, Phys. Rev. D 78, 092003 (2008).

[25] C. Robilliard, R. Battesti, M. Fouché, J. Mauchain, A.-M. Sautivet, F. Amiranoff, and C. Rizzo, "No "Light Shining Through a Wall": Results from a Photoregeneration Experiment", Phys. Rev. Lett. 99, 190403 (2007).

[26] M. Fouche et al., Search for photon oscillations into massive particles, Phys. Rev. D 78, 032013 (2008).

[27] K. Ehret et al. (ALPS Collaboration), New ALPS results on hidden-sector lightweights, Phys. Lett. B 689, 149 (2010).

[28] R. Balou et al. (OSCAR Collaboration), New exclusion limits on scalar and pseudoscalar axionlike particles from light shining through a wall, Phys. Rev. D 92, 092002 (2015).

[29] R. Cameron et al. (BFRT Collaboration), Search for nearly massless, weakly coupled particles by optical techniques, Phys. Rev. D 47, 3707 (1993).

[30] A. Cadène, P. Berceau, M. Fouché, R. Battesti, and C. Rizzo, Vacuum magnetic linear birefringence using pulsed fields: Status of the BMV experiment, Eur. Phys. J. D 68, 16 (2014).

[31] S. J. Chen, H. H. Mei, and W. T. Ni (Q\&A Collaboration), Q \& A experiment to search for vacuum dichroism, pseudoscalar-photon interaction and millicharged fermions, Mod. Phys. Lett. A 22, 2815 (2007).

[32] H. H. Mei, W. T. Ni, S. J. Chen, and S.-S. Pan (Q\&A Collaboration), Axion search with Q \& A experiment, Mod. Phys. Lett. A 25, 983 (2010).

[33] F. Della Valle, E. Milotti, A. Ejlli, G. Messineo, L. Piemontese, G. Zavattini, U. Gastaldi, R. Pengo, and G. Ruoso (PVLAS Collaboration), First results from the new PVLAS apparatus: A new limit on vacuum magnetic birefringence, Phys. Rev. D 90, 092003 (2014).

[34] V. Popov, On the experimental search for photon mixing, Turk. J. Phys. 23, 943 (1999).

[35] M. Glück, S. Rakshit, and E. Reya, Lamb shift contribution of very light charged particles, Phys. Rev. D 76, 091701 (2007).
[36] J. Jaeckel, Probing Minicharged Particles with Tests of Coulomb's Law, Phys. Rev. Lett. 103, 080402 (2009).

[37] J. Jaeckel and S. Roy, Spectroscopy as a test of Coulomb's law: A probe of the hidden sector, Phys. Rev. D 82, 125020 (2010).

[38] R. Pohl et al., The size of the proton, Nature (London) 466, 213 (2010).

[39] A. Antognini et al., Proton structure from the measurement of $2 \mathrm{~S}-2 \mathrm{P}$ transition frequencies of muonic hydrogen, Science 339, 417 (2013).

[40] R. Pohl et al., Laser spectroscopy of muonic deuterium, Science 353, 669 (2016).

[41] A. Beyer et al., The Rydberg constant and proton size from atomic hydrogen, Science 358, 79 (2017).

[42] V. Barger, Ch. W. Chiang, W. Y. Keung, and D. Marfatia, Proton Size Anomaly, Phys. Rev. Lett. 106, 153001 (2011).

[43] P. Brax and C. Burrage, Atomic precision tests and light scalar couplings, Phys. Rev. D 83, 035020 (2011).

[44] U. D. Jentschura, Light sea fermions in electron-proton and muon-proton interactions, Phys. Rev. A 88, 062514 (2013),

[45] U. D. Jentschura, Muonic bound systems, virtual particles and proton radius, Phys. Rev. A 92, 012123 (2015),

[46] Y. Sh. Liu, D. McKeen, and G. A. Miller, Electrophobic Scalar Boson and Muonic Puzzles, Phys. Rev. Lett. 117, 101801 (2016).

[47] G. 'tHooft and M. Veltman, One-loop divergencies in the theory of gravitation, Ann. Inst. Henri Poincaré, A 20, 69 (1974).

[48] K. S. Stelle, Renormalization of higher derivative quantum gravity, Phys. Rev. D 16, 953 (1977).

[49] M. H. Goroff and A. Sagnotti, Quantum gravity at two loops, Phys. Lett. 160B, 81 (1985).

[50] M. H. Goroff and A. Sagnotti, The ultraviolet behavior of Einstein gravity, Nucl. Phys. B266, 709 (1986).

[51] J.F. Donoghue, General relativity as an effective field theory: The leading quantum corrections, Phys. Rev. D 50, 3874 (1994).

[52] J. F. Donoghue, Leading Quantum Correction to the Newtonian Potential, Phys. Rev. Lett. 72, 2996 (1994).

[53] H. Gies, B. Knorr, S. Lippoldt, and F. Saueressig, Gravitational Two-Loop Counterterm is Asymptotically Safe, Phys. Rev. Lett. 116, 211302 (2016).

[54] C. Arzt, Reduced effective Lagrangians, Phys. Lett. B 342, 189 (1995).

[55] C. Grosse-Knetter, Effective Lagrangians with higher derivatives and equations of motion, Phys. Rev. D 49, 6709 (1994).

[56] H. Georgi, On-shell effective field theory, Nucl. Phys. B361, 339 (1991).

[57] M. B. Einhorn and J. Wudka, Effective $\beta$-functions for effective field theory, J. High Energy Phys. 08 (2001) 025.

[58] S. Weinberg, Phenomenological Lagrangians, Physica (Amsterdam) 96A, 327 (1979).

[59] J. Gasser and H. Leutwyler, Chiral perturbation theory to one loop, Ann. Phys. (N.Y.) 158, 142 (1984). 
[60] J. Gasser and H. Leutwyler, Chiral perturbation theory: Expansions in the mass of the strange quark, Nucl. Phys. B250, 465 (1985).

[61] G. Ecker, Low-energy QCD, Prog. Part. Nucl. Phys. 36, 71 (1996).

[62] J. Halter, An effective Lagrangian for photons, Phys. Lett. B 316, 155 (1993).

[63] X. W. Kong and F. Ravndal, Quantum corrections to the QED vacuum energy, Nucl. Phys. B526, 627 (1998).

[64] D. A. Dicus, C. Kao, and W. W. Repko, Effective Lagrangians and low-energy photon-photon scattering, Phys. Rev. D 57, 2443 (1998).

[65] M. D. Schwartz, Quantum Field Theory and the Standard Model (Cambridge University Press, Cambridge, England, 2014).

[66] A. Eichhorn, H. Gies, and D. Roscher, Renormalization flow of axion electrodynamics, Phys. Rev. D 86, 125014 (2012).

[67] B. Grinstein, D. O'Connel, and M. B. Wise, The Lee-Wick standard model, Phys. Rev. D 77, 025012 (2008).

[68] A. Accioly and M. Dias, Algorithm for probing the unitarity of topologically massive models, Int. J. Theor. Phys. 44, 1123 (2005).

[69] P. Podolsky, A generalized electrodynamics Part I: Non-quantum, Phys. Rev. 62, 68 (1942).

[70] P. Podolsky and C. Kikuchi, A generalized electrodynamics Part II: Quantum, Phys. Rev. 65, 228 (1944).

[71] A. Pais and G. E. Uhlenbeck, On field theories with non-localized action, Phys. Rev. 79, 145 (1950).

[72] C. A. P. Galvão and B. M. Pimentel, The canonical structure of Podolsky generalized electrodynamics, Can. J. Phys. 66, 460 (1988).

[73] A. Accioly and M. Dias, Boson-boson effective nonrelativistic potential for higher-derivative electromagnetic theories in D dimensions, Phys. Rev. D 70, 107705 (2004).

[74] Z. Haghani, T. Harko, H. R. Sepangi, and S. Shahidi, Vector dark energy models with quadratic terms in the Maxwell tensor derivatives, Eur. Phys. J. C 77, 137 (2017).

[75] T. D. Lee and G. C. Wick, Negative metric and the unitarity of the S matrix, Nucl. Phys. B9, 209 (1969).

[76] T.D. Lee and G.C. Wick, Finite theory of quantum electrodynamics, Phys. Rev. D 2, 1033 (1970).

[77] R. E. Cutkosky, P. V. Landshoff, D. I. Olive, and J. C. Polkinghorne, A non-analytic S matrix, Nucl. Phys. B12, 281 (1969).

[78] S. Weinberg, The Quantum Theory of Fields. Vol. 1: Foundations (Cambridge University Press, Cambridge, England, 1995).

[79] H. Gies and J. Jaeckel, Tunneling of the 3rd kind, J. High Energy Phys. 08 (2009) 063.

[80] S. Villalba-Chávez, A. E. Shabad, and C. Müller, Criticality and dominance of axion physics in highly magnetized vacuum (in preparation).

[81] G. G. di Cortona, E. Hardy, J. P. Vega, and G. Villadoro, The QCD axion, precisely, J. High Energy Phys. 01 (2016) 034.
[82] M. Giannotti, I. G. Irastorza, J. Redondo, A. Ringwald, and K. Saikawa, Stellar recipes for axion hunters, J. Cosmol. Astropart. Phys. 10 (2017) 010.

[83] I. G. Irastorza and J. Redondo, New experimental approaches in the search for axion-like particles, Prog. Part. Nucl. Phys. 102, 89 (2018).

[84] B. M. Dillon and B. King, ALP production through nonlinear Compton scattering in intense fields, Eur. Phys. J. C 78, 775 (2018).

[85] E. Gabrielli, K. Huitu, and S. Roy, Photon propagation in magnetic and electric fields with scalar/pseudoscalar couplings: A new look, Phys. Rev. D 74, 073002 (2006).

[86] M. E. Peskin and D. V. Schroeder, An Introduction to Quantum Field Theory, (Perseus Books, Massachusetts, 1995).

[87] I. S. Gradshteyn and I. M. Ryzhik, Table of Integrals, Series and Products, 7th ed. (Elsevier, New York, 2007).

[88] B. Döbrich, J. Jaeckel, F. Kahlhoefer, A. Ringwald, and K. Schmidt-Hoberg, ALPtraum: ALP production in proton beam dump experiment, J. High Energy Phys. 02 (2016) 018 .

[89] J. Jaeckel and M. Spannowsky, Probing MeV to $90 \mathrm{GeV}$ axion-like particles with LEP and LHC, Phys. Lett. B 753, 482 (2016).

[90] M. Arik et al., Search for Sub-eV Mass Solar Axions by the CERN Axion Solar Telescope with 3He Buffer Gas, Phys. Rev. Lett. 107, 261302 (2011).

[91] S. Villalba-Chávez, T. Podszus, and C. Müller, Polarizationoperator approach to optical signatures of axion-like particles in strong laser pulses, Phys. Lett. B 769, 233 (2017).

[92] J. T. Mendonça, Axion excitation by intense laser fields, Eurphys. Lett. 79, 21001 (2007).

[93] H. Gies, Strong laser fields as a probe for fundamental physics, Eur. Phys. J. D 55, 311 (2009).

[94] B. Döbrich and H. Gies, Axion-like-particle search with high-intensity lasers, J. High Energy Phys. 10 (2010) 022.

[95] A. Di Piazza, C. Müller, K. Z. Hatsagortsyan, and C. H. Keitel, Extremely high-intensity laser interactions with fundamental quantum systems, Rev. Mod. Phys. 84, 1177 (2012).

[96] S. Villalba-Chávez and C. Müller, Searching for minicharged particles via birefringence, dichroism and Raman spectroscopy of the vacuum polarized by a high-intensity laser wave, Ann. Phys. (Amsterdam) 339, 460 (2013).

[97] S. Villalba-Chávez and A. Di Piazza, Axion-induced birefringence effects in laser driven nonlinear vacuum interaction, J. High Energy Phys. 11 (2013) 136.

[98] S. Villalba-Chávez, Laser-driven search of axion-like particles including vacuum polarization effects, Nucl. Phys. B881, 1 (2014).

[99] S. Villalba-Chávez and C. Müller, Light dark matter candidates in intense laser pulses I: Paraphotons and fermionic minicharged particles, J. High Energy Phys. 06 (2015) 177.

[100] S. Villalba-Chávez and C. Müller, Light dark matter candidates in intense laser pulses II: The relevance of the spin degrees of freedom, J. High Energy Phys. 02 (2016) 027. 
[101] S. Villalba-Chávez, S. Meuren, and C. Müller, Minicharged particles search by strong laser pulse-induced vacuum polarization effects, Phys. Lett. B 763, 445 (2016).

[102] R. Bufalo, B. M. Pimentel, and G. E. R. Zambrano, Path integral quantization of generalized quantum electrodynamics, Phys. Rev. D 83, 045007 (2011).

[103] R. Bufalo, B. M. Pimentel, and G. E. R. Zambrano, Renormalizability of generalized quantum electrodynamics, Phys. Rev. D 86, 125023 (2012).

[104] P. Van Nieuwenhuizen, On ghost-free tensor Lagrangians and linearized gravitation, Nucl. Phys. B60, 478 (1973).

[105] E. Sezgin and P. Van Nieuwenhuizen, New ghost-free gravity Lagrangians with propagating torsion, Phys. Rev. D 21, 3269 (1980).
[106] M. Giannotti and E. Mottola, Trace anomaly and massless scalar degrees of freedom in gravity, Phys. Rev. D 79, 045014 (2009).

[107] L. B. Okun, Limits of electrodynamics: Paraphotons?, Sov. Phys. JETP 56, 502 (1982).

[108] J. C. Maxwell, A Treatise on Electricity and Magnetism (Dover Publications, New York, 1954).

[109] S. J. Plimpton and W. E. Lawton, A very accurate test of Coulomb's law of force between charges, Phys. Rev. 50, 1066 (1936).

[110] E. R. Williams, J.E. Faller, and H. A. Hill, New Experimental Test of Coulomb's Law: A Laboratory Upper Limit on the Photon Rest Mass, Phys. Rev. Lett. 26, 721 (1971). 